\title{
DISTANCE BETWEEN UNITARY ORBITS IN VON NEUMANN ALGEBRAS
}

\author{
Fumio Hiai and Yoshiniro NaKamura \\ Dedicated to Professor Shozo Koshi on his 60th birthday
}

\begin{abstract}
Let $\mathscr{M}$ be a semifinite factor. For normal operators $x$ and $y$ in $\mathscr{M}$, introducing the spectral distance $\delta(x, y)$, we show that $\delta(x, y) \geq$ $\operatorname{dist}(\mathscr{U}(x), \mathscr{U}(y)) \geq c^{-1} \delta(x, y)$ with a universal constant $c$, where $\operatorname{dist}(\mathscr{U}(x), \mathscr{U}(y))$ denotes the distance between the unitary orbits $\mathscr{U}(x)$ and $\mathscr{U}(y)$. The equality $\operatorname{dist}(\mathscr{U}(x), \mathscr{U}(y))=\delta(x, y)$ holds in several cases. Submajorizations are established concerning the spectral scales of $\tau$-measurable selfadjoint operators affiliated with $\mathscr{M}$. Using these submajorizations, we obtain the formulas of $L^{p}$ distance and anti- $L^{p}$-distance between unitary orbits of $\tau$-measurable selfadjoint operators in terms of their spectral scales. Furthermore the formulas of those distances in Haagerup $L^{p}$-spaces are obtained when $\mathscr{M}$ is a type $\mathrm{III}_{1}$ factor. The appendix by $\mathrm{H}$. Kosaki is the generalized Powers-Størmer inequality in Haagerup $L^{p}$-spaces.
\end{abstract}

Introduction. It is an interesting problem in matrix theory to estimate distances between unitary orbits of matrices by their eigenvalues. Let $A$ and $B$ be $n \times n$ normal matrices whose eigenvalues are $\alpha_{1}, \ldots, \alpha_{n}$ and $\beta_{1}, \ldots, \beta_{n}$, respectively, with multiplicities counted. Let $\operatorname{dist}(\mathscr{U}(A), \mathscr{U}(B))$ denote the distance between the unitary orbits $\mathscr{U}(A)$ and $\mathscr{U}(B)$. The optimal matching distance between the eigenvalues of $A$ and $B$ is given by

$$
\delta(A, B)=\min _{\pi} \max _{i}\left|\alpha_{i}-\beta_{\pi(i)}\right|,
$$

where $\pi$ runs over all permutations of $\{1, \ldots, n\}$. Then

$$
\operatorname{dist}(\mathscr{U}(A), \mathscr{U}(B)) \leq \delta(A, B)
$$

is immediate. Bhatia, Davis and McIntosh [9] proved that

$$
\operatorname{dist}(\mathscr{U}(A), \mathscr{U}(B)) \geq c^{-1} \delta(A, B)
$$

with a universal constant $c$. A difficult and still open conjecture is that $\operatorname{dist}(\mathscr{U}(A), \mathscr{U}(B))=\delta(A, B)$ holds for every pair of normal matrices $A$ and $B$ (i.e. $c=1$ ). But this equality was proved to hold for several classes of normal matrices (see $[7,10,21,41,45]$ ). The analogous 
results were obtained also in the infinite dimensional case by introducing the spectral distance $\delta(A, B)$ for normal operators $A$ and $B$ on a Hilbert space (see $[6,14])$.

From the viewpoint of von Neumann algebras, the results stated above are concerned with the case of factors of type I. The aim of this paper is to study distances between unitary orbits of operators in more general von Neumann algebras. In most results of this paper except $\S 5, \mathscr{M}$ is a semifinite factor. Let $\mathscr{M}$ be a semifinite von Neumann algebra with a fixed faithful normal semifinite trace $\tau$. Let $\mathscr{U}$ be the set of all unitaries in $\mathscr{M}$ and $\mathscr{U}(x)$ the unitary orbit $\left\{u x u^{*}: u \in \mathscr{U}\right\}$ of $x \in \mathscr{M}$. In $\S 1$ of this paper, for normal operators $x$ and $y$ in $\mathscr{M}$, we introduce the spectral distance $\delta(x, y)$ by comparing the traces of spectral projections of $x$ and $y$. This $\delta(x, y)$ extends the optimal matching distance given above for normal matrices. When $\mathscr{M}$ is a $\sigma$-finite semifinite factor, we show that

$$
\delta(x, y) \geq \operatorname{dist}(\mathscr{U}(x), \mathscr{U}(y)) \geq c^{-1} \delta(x, y)
$$

for all normal elements $x, y \in \mathscr{M}$ where $c$ is a universal constant given in [9]. As was shown in [14] for the type I case, this second inequality is an immediate consequence of a powerful result of [9]. On the other hand, we give a variant of the marriage theorem in order to prove the first inequality. Section 1 contains also a result on distances between unitary orbits in the type III case. In $\S 2$, we establish the equality $\operatorname{dist}(\mathscr{U}(x), \mathscr{U}(y))=\delta(x, y)$ for several classes of $x$ and $y$ corresponding to the known classes of matrices.

Several (sub)majorizations are known for the eigenvalues and the singular values of matrices. The Lidskii-Wielandt theorem is especially famous and important, which gives a useful device in deriving various norm inequalities for matrices. See $[1,31,32]$ for majorization theory on matrices and compact operators. The noncommutative integration theory (in the semifinite case) was founded in $[15,39,44]$. The concept of $\tau$-measurable operators introduced in [34] gives a nice foundation of noncommutative $L^{p}$-spaces $L^{p}(\mathscr{M})$. The majorization theory in semifinite von Neumann algebras was recently developed in $[22-24,26,27,33]$ by using the notion of generalized $s$-numbers or spectral scales of $\tau$-measurable operators. In particular, we have generalized in [24] the Lidskii-Wielandt theorem to the majorizations for the spectral scales of selfadjoint operators in the space $L^{1}(\mathscr{M})$ when $\tau(1)<\infty$.

We denote by $\tilde{\mathscr{M}}_{s a}$ the set of all $\tau$-measurable selfadjoint operators affiliated with $\mathscr{M}$. When $\tau(1)<\infty$, for $x \in \tilde{\mathscr{M}}_{s a}$ with the spectral 
decomposition $x=\int_{-\infty}^{\infty} s d e_{s}$, the spectral scale $\lambda(x)$ of $x$ is the function on $(0, \tau(1))$ defined by $\lambda_{t}(x)=\inf \left\{s \in \mathbf{R}: \tau\left(e_{s}^{\perp}\right) \leq t\right\}$ where $e_{s}^{\perp}=1-e_{s}$. When $\tau(1)=\infty$, we define the spectral scale of $x \in \tilde{\mathscr{M}}_{s a}$ in some modification. In $\S 3$, for $x, y \in \tilde{\mathscr{M}}_{s a}$ when $\tau(1)<\infty$, working on the majorizations in [24] we show that $|\lambda(x)-\lambda(y)|$ is submajorized by $|\lambda(x-y)|$ and the latter is submajorized by $|\lambda(x)-\check{\lambda}(y)|$ where $\grave{\lambda}(y)=-\lambda(-y)$. In $\S 4$, by use of these submajorizations and the notion of spectral equivalence, we obtain the following formulas of $L^{p}$-distance and anti- $L^{p}$-distance between unitary orbits: when $\mathscr{M}$ is a finite factor, for $x, y \in \tilde{\mathscr{M}}_{s a}$ and $1 \leq p \leq \infty$

$$
\begin{aligned}
& \inf _{u \in \mathscr{U}}\left\|x-u y u^{*}\right\|_{p}=\|\lambda(x)-\lambda(y)\|_{p}, \\
& \sup _{u \in \mathscr{U}}\left\|x-u y u^{*}\right\|_{p}=\|\lambda(x)-\check{\lambda}(y)\|_{p} .
\end{aligned}
$$

When $\mathscr{M}$ is infinite semifinite, the analogous submajorizations and $L^{p}$-distances of unitary orbits are obtained for $x$ and $y$ in a certain subclass of $\tilde{\mathscr{M}}_{s a}$ with the modified spectral scales. Furthermore those $L^{p}$-distances for $\tau$-measurable selfadjoint $x$ and skew-adjoint $y$ are estimated in terms of their spectral scales by the majorization method of [3].

Finally in $\S 5$, we discuss distances between unitary orbits in Haagerup $L^{p}$-spaces $L^{p}(\mathscr{M})$ introduced in [19] (also [42]). When $\mathscr{M}$ is a factor of type $\mathrm{III}_{1}$, we exactly estimate the $L^{p}$-distance and the anti$L^{p}$-distance between unitary orbits of selfadjoint elements in $L^{p}(\mathscr{M})$ by using the homogeneity of type III $_{1}$ factors [13] and the generalized Powers-Størmer inequality by H. Kosaki. Also, when $\mathscr{M}$ is an arbitrary infinite factor, the formulas of $L^{p}$-distances are obtained for some special classes of elements in $L^{p}(\mathscr{M})$.

This paper contains the appendix by $\mathrm{H}$. Kosaki where the PowersStørmer inequality is generalized to positive elements in Haagerup $L^{p}$-spaces. For this sake, his appendix also generalizes an inequality due to Ando [2] as follows:

$$
\begin{aligned}
\int_{0}^{s} \mu_{t}(f(a)-f(b)) d t & \leq \int_{0}^{s} \mu_{t}(f(|a-b|)) d t \\
& =\int_{0}^{s} f\left(\mu_{t}(a-b)\right) d t, \quad s>0,
\end{aligned}
$$

for positive $\tau$-measurable operators $a, b$ affiliated with a semifinite von Neumann algebra, where $\mu_{t}(\cdot)$ denotes the generalized $s$-number and $f$ is any operator monotone function on $[0, \infty)$ with $f(0)=0$. This inequality is of considerable importance in majorization theory. 
1. Distance between unitary orbits of normal operators. Let $\mathscr{M}$ be a von Neumann algebra on a Hilbert space $\mathscr{H}$ and $\mathscr{U}$ the set of all unitaries in $\mathscr{M}$. For each $x \in \mathscr{M}$, we denote by $\mathscr{U}(x)$ the unitary orbit $\left\{u x u^{*}: u \in \mathscr{U}\right\}$ of $x$ and by $\sigma(x)$ the spectrum of $x$. For $x, y \in \mathscr{M}$, let $\operatorname{dist}(\mathscr{U}(x), \mathscr{U}(y))$ be the distance between $\mathscr{U}(x)$ and $\mathscr{U}(y)$, i.e.

$$
\operatorname{dist}(\mathscr{U}(x), \mathscr{U}(y))=\inf _{u \in \mathscr{U}}\left\|x-u y u^{*}\right\|,
$$

and $h(\sigma(x), \sigma(y))$ the Hausdorff distance between $\sigma(x)$ and $\sigma(y)$, i.e.

$$
h(\sigma(x), \sigma(y))=\max \left\{\sup _{\alpha \in \sigma(x)} \operatorname{dist}(\alpha, \sigma(y)), \sup _{\beta \in \sigma(y)} \operatorname{dist}(\beta, \sigma(x))\right\} .
$$

It is known (see [14, Proposition 2.1]) that if $x$ and $y$ are normal operators in $\mathscr{M}$, then

$$
\operatorname{dist}(\mathscr{U}(x), \mathscr{U}(y)) \geq h(\sigma(x), \sigma(y)) .
$$

In what follows except in $\S 5$, unless otherwise stated, let $\mathscr{M}$ be a semifinite von Neumann algebra with a faithful normal semifinite trace $\tau$. For a normal operator $x$ in $\mathscr{M}$ and a Borel subset $E$ of $\mathbf{C}$, let $e_{E}(x)$ denote the spectral projection of $x$ corresponding to $E$. Also let $E_{r}=\{\alpha \in \mathbf{C}$ : $\operatorname{dist}(\alpha, E)<r\}$ for $r>0\left(\varnothing_{r}=\varnothing\right)$. Given two normal elements $x, y \in \mathscr{M}$, we now define the spectral distance $\delta(x, y)$ as follows: $\delta(x, y)$ is the infimum of $r>0$ such that $\tau\left(e_{V}(x)\right) \leq \tau\left(e_{V_{r}}(y)\right)$ and $\tau\left(e_{V}(y)\right) \leq \tau\left(e_{V_{r}}(x)\right)$ for every open subset $V$ of $\mathbf{C}$. In particular when $\mathscr{M}$ is the algebra $\mathbf{M}_{n}$ of all $n \times n$ complex matrices, it follows from the marriage theorem [20] that $\delta(x, y)$ coincides with the optimal matching distance, that is,

$$
\delta(x, y)=\min _{\pi} \max _{1 \leq i \leq n}\left|\alpha_{i}-\beta_{\pi(i)}\right|
$$

where $\alpha_{1}, \ldots, \alpha_{n}$ and $\beta_{1}, \ldots, \beta_{n}$ are the eigenvalues of $x$ and $y$, respectively, with multiplicities counted and $\pi$ runs over all permutations of $\{1, \ldots, n\}$.

The purpose of this section is to estimate $\operatorname{dist}(\mathscr{U}(x), \mathscr{U}(y))$ in terms of $\delta(x, y)$ for normal elements $x, y \in \mathscr{M}$. We begin with the following theorem which can be shown as in the proof of [14, Theorem 2.4] by appealing to [9, Theorem 4.2].

THEOREM 1.1. If $x$ and $y$ are normal operators in $\mathscr{M}$, then

$$
\operatorname{dist}(\mathscr{U}(x), \mathscr{U}(y)) \geq c^{-1} \delta(x, y)
$$

where $c$ is a universal constant. 
For $c$ in the theorem, an upper bound given in [8] is

$$
(\pi / 2) \int_{0}^{\pi} t^{-1} \sin t d t \quad(<2.91)
$$

which is at present the best estimate even when $\mathscr{M}=\mathbf{M}_{n}$.

To obtain the converse estimation, we need the next lemma which is a variant of the marriage theorem.

Lemma 1.2. Let $\left\{a_{1}, \ldots, a_{m}\right\}$ and $\left\{b_{1}, \ldots, b_{n}\right\}$ be finite sets in $[0, \infty]$ and $R$ a subset of $\{1, \ldots, m\} \times\{1, \ldots, n\}$. Consider the following conditions (1) and (2):

(1) $\sum_{i \in A} a_{i} \leq \sum_{j \in R_{A}} b_{j}$ for every $A \subseteq\{1, \ldots, m\}$ where $R_{A}=$ $\bigcup_{i \in A} R_{i}, R_{l}=\{j:(i, j) \in R\}$,

(2) $\sum_{j \in B} b_{j} \leq \sum_{i \in R^{B}} a_{i}$ for every $B \subseteq\{1, \ldots, n\}$ where $R^{B}=$ $\bigcup_{j \in B} R^{j}, R^{j}=\{i:(i, j) \in R\}$.

If both (1) and (2) hold, or if (1) holds and $\sum_{i=1}^{m} a_{i}=\sum_{j=1}^{n} b_{j}$ $<\infty$, then there exist $c_{i j} \in[0, \infty], 1 \leq i \leq m, 1 \leq j \leq n$, such that

(i) $c_{i j}=0$ unless $(i, j) \in R$,

(ii) $\sum_{j=1}^{n} c_{i j}=a_{i}$ for all $i$,

(iii) $\sum_{i=1}^{m} c_{i j}=b_{j}$ for all $j$.

Furthermore if all $a_{i}$ and $b_{j}$ are in $\{0,1,2, \ldots, \infty\}$, then all $c_{i j}$ are taken in $\{0,1,2, \ldots, \infty\}$.

Proof. First assume (1) alone and show the existence of $c_{i j} \in[0, \infty]$ satisfying (i), (ii) and

(iii') $\sum_{i=1}^{m} c_{i j} \leq b_{j}$ for all $j$.

For any $i$ with $a_{i}=\infty$, there is a $j_{1} \in R_{i}$ with $b_{j_{1}}=\infty$, so let $c_{i j_{1}}=\infty$ and $c_{i j}=0$ for $j \neq j_{1}$. Moreover, for any $i$ with $a_{i}=0$, let $c_{i j}=0$ for all $j$. Hence it suffices to consider the case when $a_{i} \in(0, \infty)$ for all $i$. In this case, removing $b_{j}=0$ and replacing $b_{j}=\infty$ by a number large enough, we may assume also that $b_{j} \in(0, \infty)$ for all $j$. For each sufficiently large natural number $N$, let $k_{i}$ (resp. $l_{j}$ ) be the largest (resp. smallest) natural number such that $k_{i} / N \leq a_{i}$ (resp. $l_{j} / N \geq b_{j}$ ). Take mutually disjoint sets $\mathscr{A}_{1}, \ldots, \mathscr{A}_{m}$ and $\mathscr{B}_{1}, \ldots, \mathscr{B}_{n}$ with $\left|\mathscr{A}_{i}\right|=k_{i}$ and $\left|\mathscr{B}_{j}\right|=l_{j}$ where $|\cdot|$ denotes the cardinality. Let $\mathscr{A}_{0}=\bigcup_{i=1}^{m} \mathscr{A}_{i}, \mathscr{B}_{0}=\bigcup_{j=1}^{n} \mathscr{B}_{j}$ and $\mathscr{R}$ be the set of all $(\alpha, \beta) \in \mathscr{A}_{0} \times \mathscr{B}_{0}$ such that $\alpha \in \mathscr{A}_{i}$ and $\beta \in \mathscr{B}_{j}$ for some $(i, j) \in R$. For every $\mathscr{A} \subseteq \mathscr{A}_{0}$, letting $A=\left\{i: \mathscr{A} \cap \mathscr{A}_{i} \neq \varnothing\right\}$, we have

$$
|\mathscr{A}| \leq \sum_{i \in A} k_{i} \leq N \sum_{i \in A} a_{i} \leq N \sum_{j \in R^{4}} b_{j} \leq \sum_{j \in R^{4}} l_{j}=\left|\mathscr{R}^{\mathscr{A}}\right|
$$


since $\mathscr{R}^{\mathscr{A}}=\bigcup_{j \in R^{A}} \mathscr{B}$. Hence, by the usual marriage theorem [20], there exists an injective map $\Phi: \mathscr{A}_{0} \rightarrow \mathscr{B}_{0}$ such that $(\alpha, \Phi(\alpha)) \in \mathscr{R}$ for all $\alpha \in \mathscr{A}_{0}$. Define

$$
c_{i j}^{(N)}=N^{-1}\left|\left\{\alpha \in \mathscr{A}_{0}:(\alpha, \Phi(\alpha)) \in \mathscr{A}_{i} \times \mathscr{B}_{j}\right\}\right|, \quad 1 \leq i \leq m, 1 \leq j \leq n .
$$

Then $c_{i j}^{(N)}=0$ unless $(i, j) \in R, \sum_{j=1}^{n} c_{i j}^{(N)}=k_{i} / N$ and $\sum_{i=1}^{m} c_{i j}^{(N)} \leq$ $l_{j} / N$. Since $\sum_{i, j} c_{i j}^{(N)} \leq \sum_{i=1}^{m} a_{i}<\infty$, we can choose a cluster point $\left(c_{i j}\right)$ of a sequence $\left\{\left(c_{i j}^{(N)}\right)\right\}$ in $\mathbf{R}^{m n}$, which satisfies (i), (ii), and (iii').

If $\sum_{i=1}^{m} a_{i}=\sum_{j=1}^{n} b_{j}<\infty$, then the above $\left(c_{i j}\right)$ automatically satisfies (iii) because $\sum_{j=1}^{n}\left(\sum_{i=1}^{m} c_{i j}\right)=\sum_{j=1}^{n} b_{j}<\infty$. Now assume (2) as well. Let $A_{0}=\left\{i: a_{i}<\infty\right\}$ and

$$
B_{0}=\left\{j: b_{j}<\infty \text { and } a_{i}<\infty \text { for all } i \in R^{j}\right\} .
$$

Denote by $\Gamma$ the set of all $\left(d_{i j}: i \in A_{0}, 1 \leq j \leq n\right)$ in $\mathbf{R}^{\left|A_{0}\right| n}$ such that $d_{i j} \geq 0, d_{i j}=0$ unless $(i, j) \in R, \sum_{j=1}^{n} d_{i j}=a_{i}$ for all $i \in A_{0}$ and $\sum_{i \in A_{0}} d_{i j} \leq b_{j}$ for all $1 \leq j \leq n$. Because $\Gamma$ is a bounded closed subset of $\mathbf{R}^{\left|A_{0}\right| n}$ which is nonempty from the first argument, we can choose a $\left(c_{i j}\right) \in \Gamma$ such that

$$
\sum_{i \in A_{0}} \sum_{j \in B_{0}} c_{i j}=\sup \left\{\sum_{i \in A_{0}} \sum_{j \in B_{0}} d_{i j}:\left(d_{i j}\right) \in \Gamma\right\} .
$$

Suppose $\sum_{i \in A_{0}} c_{i j_{0}}<b_{j_{0}}$ for some $j_{0} \in B_{0}$. Since $R^{B_{0}} \subseteq A_{0}$, we get

$$
\sum_{i \in A_{0}} \sum_{j \in B_{0}} c_{i j}<\sum_{j \in B_{0}} b_{j} \leq \sum_{i \in A_{0}} a_{i}=\sum_{i \in A_{0}} \sum_{j=1}^{n} c_{i j},
$$

so that $c_{i_{0} j_{0}^{\prime}}>0$ for some $i_{0} \in A_{0}$ and $j_{0}^{\prime} \notin B_{0}$. If $\tilde{c}_{i_{0} j_{0}}=c_{i_{0} j_{0}}+c$ and $\tilde{c}_{i_{0} j_{0}^{\prime}}=c_{i_{0} j_{0}^{\prime}}-c$ with a sufficiently small $c>0$ and if $\tilde{c}_{i j}=c_{i j}$ for other $(i, j)$, then $\left(\tilde{c}_{i j}\right) \in \Gamma$. This is a contradiction, so that $\sum_{i \in A_{0}} c_{i j}=b_{j}$ for all $j \in B_{0}$. For any $i \notin A_{0}$, there is a $j_{1} \in R_{i}$ with $b_{j_{1}}=\infty$, so let $c_{i j_{1}}=\infty$. For any $j \notin B_{0}$, there is an $i_{1} \in R^{j} \backslash A_{0}$ with $a_{i_{1}}=\infty$, so let $c_{i_{1} j}=b_{j}-\sum_{i \in A_{0}} c_{i j}$. Finally let $c_{i j}=0$ for other $(i, j)$ with $i \notin A_{0}$ and $1 \leq j \leq n$. Thus we obtain $c_{i j}$ satisfying (i)-(iii).

The last part of the lemma is readily seen from the above proof.

THeORem 1.3. Assume that $\mathscr{M}$ is a finite factor or $\mathscr{M}$ is nonatomic with $\tau(1)<\infty$. If $x$ and $y$ are normal operators in $\mathscr{M}$, then $\delta(x, y)$ is equal to the infimum of $r>0$ such that $\tau\left(e_{V}(x)\right) \leq \tau\left(e_{V_{r}}(y)\right)$ for every open set $V \subseteq \mathbf{C}$. 
Proof. For normal elements $x, y \in \mathscr{M}$, let $\delta_{0}(x, y)$ be the infimum given in the theorem. Obviously $\delta_{0}(x, y) \leq \delta(x, y)$. It is immediate that $\delta_{0}(x, y)$ as well as $\delta(x, y)$ satisfies the triangle inequality. For any $\varepsilon>0$, take normal operators $x^{\prime}$ and $y^{\prime}$ in $\mathscr{M}$ with finite spectra such that $\left\|x^{\prime}-x\right\|<\varepsilon$ and $\left\|y^{\prime}-y\right\|<\varepsilon$. Then

$$
\delta_{0}\left(x^{\prime}, y^{\prime}\right) \leq \delta_{0}\left(x^{\prime}, x\right)+\delta_{0}(x, y)+\delta_{0}\left(y, y^{\prime}\right) \leq \delta_{0}(x, y)+2 c \varepsilon
$$

by Theorem 1.1, and similarly

$$
\delta(x, y) \leq \delta\left(x^{\prime}, y^{\prime}\right)+2 c \varepsilon .
$$

Let $r>\delta_{0}\left(x^{\prime}, y^{\prime}\right)$. Writing $x^{\prime}=\sum_{i=1}^{m} \alpha_{i} p_{i}$ and $y^{\prime}=\sum_{j=1}^{n} \beta_{j} q_{j}$ where $\sum_{i=1}^{m} p_{i}=\sum_{j=1}^{n} q_{j}=1$, we define $a_{i}=\tau\left(p_{i}\right), b_{j}=\tau\left(q_{j}\right)$ and $R=$ $\left\{(i, j):\left|\alpha_{i}-\beta_{j}\right| \leq r\right\}$. Then (1) in Lemma 1.2 holds and $\sum_{i=1}^{m} a_{i}=$ $\sum_{j=1}^{n} b_{j}<\infty$. By Lemma 1.2, there are $c_{i j} \in[0, \infty), 1 \leq i \leq m, 1 \leq$ $j \leq n$, satisfying (i)-(iii). When $\mathscr{M}=\mathbf{M}_{n}$ (with the usual trace $\tau$ ), all $c_{i j}$ are integers. Otherwise $\mathscr{M}$ is nonatomic. In either case, $p_{i}$ and $q_{j}$ are divided into mutually orthogonal projections as follows: $p_{i}=\sum_{j=1}^{n} p_{i j}$ and $q_{j}=\sum_{i=1}^{m} q_{i j}$ with $\tau\left(p_{i j}\right)=\tau\left(q_{i j}\right)=c_{i j}$. Hence $x^{\prime}=\sum_{i, j} \alpha_{i} p_{i j}, y^{\prime}=\sum_{i, j} \beta_{j} q_{i j}$ and $\left|\alpha_{i}-\beta_{j}\right| \leq r$ if $p_{i j} \neq 0$. This shows $\delta\left(x^{\prime}, y^{\prime}\right) \leq r$. Thus $\delta\left(x^{\prime}, y^{\prime}\right) \leq \delta_{0}\left(x^{\prime}, y^{\prime}\right)$, so that $\delta(x, y) \leq$ $\delta_{0}(x, y)+4 c \varepsilon$, implying $\delta(x, y)=\delta_{0}(x, y)$.

THEOREM 1.4. Assume that $\mathscr{M}$ is a $\sigma$-finite semifinite factor. Then

$$
\operatorname{dist}(\mathscr{U}(x), \mathscr{U}(y)) \leq \delta(x, y)
$$

for every pair of normal operators $x, y \in \mathscr{M}$.

Proof. For any $\varepsilon>0$, take $x^{\prime}=\sum_{i=1}^{m} \alpha_{i} p_{i}$ and $y^{\prime}=\sum_{j=1}^{n} \beta_{j} q_{j}$ as in the proof of Theorem 1.3. Let $r>\delta\left(x^{\prime}, y^{\prime}\right)$. Then (1) and (2) in Lemma 1.2 hold for $a_{i}=\tau\left(p_{i}\right), b_{j}=\tau\left(q_{j}\right)$ and $R=\left\{(i, j):\left|\alpha_{i}-\beta_{j}\right| \leq\right.$ $r\}$. Hence, using Lemma 1.2 and arguing as in the proof of Theorem 1.3 , we can write $x^{\prime}=\sum_{i, j} \alpha_{i} p_{i j}$ and $y^{\prime}=\sum_{i, j} \beta_{j} q_{i j}$ where $\tau\left(p_{i j}\right)=$ $\tau\left(q_{i j}\right)$ and $\left|\alpha_{i}-\beta_{j}\right| \leq r$ if $p_{i j} \neq 0$. It follows from the assumption of $\mathscr{M}$ that $p_{i j} \sim q_{i j}$ in the Murray-von Neumann sense for all $(i, j)$. So there exists a $u \in \mathscr{U}$ such that $p_{i j}=u q_{i j} u^{*}$ for all $(i, j)$ and hence

$$
\left\|x^{\prime}-u y^{\prime} u^{*}\right\|=\left\|\sum_{i, j}\left(\alpha_{i}-\beta_{j}\right) p_{i j}\right\| \leq r,
$$

implying $\operatorname{dist}\left(\mathscr{U}\left(x^{\prime}\right), \mathscr{U}\left(y^{\prime}\right)\right) \leq \delta\left(x^{\prime}, y^{\prime}\right)$. Therefore

$$
\begin{gathered}
\operatorname{dist}(\mathscr{U}(x), \mathscr{U}(y)) \leq \operatorname{dist}\left(\mathscr{U}\left(x^{\prime}\right), \mathscr{U}\left(y^{\prime}\right)\right)+2 \varepsilon \\
\leq \delta\left(x^{\prime}, y^{\prime}\right)+2 \varepsilon \leq \delta(x, y)+2 c \varepsilon+2 \varepsilon
\end{gathered}
$$

by Theorem 1.1, so that we get the desired inequality. 
In Theorem 1.4, the assumption of $\sigma$-finiteness of $\mathscr{M}$ cannot be removed. For instance, let $\mathscr{M}=\mathbf{B}(\mathscr{H})$, the algebra of all bounded operators on $\mathscr{H}$. When $\mathscr{H}$ is not separable, there are projections $p$ and $q$ in $\mathscr{M}$ such that $\delta(p, q)=0$ but $\operatorname{dist}(\mathscr{U}(p), \mathscr{U}(q))=1$.

The next theorem asserts that the computation of $\operatorname{dist}(\mathscr{U}(x), \mathscr{U}(y))$ is very simple in the purely infinite case. For normal elements $x, y \in$ $\mathscr{M}$, the Hausdorff distance $h(\sigma(x), \sigma(y))$ is nothing but $\delta(x, y)$ where $\tau(0)=0$ and $\tau(e)=\infty$ for each nonzero projection $e$ in $\mathscr{M}$.

THeOREM 1.5. Assume that $\mathscr{M}$ is a $\sigma$-finite factor of type III. Then

$$
\operatorname{dist}(\mathscr{U}(x), \mathscr{U}(y))=h(\sigma(x), \sigma(y))
$$

for every pair of normal operators $x, y \in \mathscr{M}$.

Proof. Since $\operatorname{dist}(\mathscr{U}(x), \mathscr{U}(y)) \geq h(\sigma(x), \sigma(y))$ as noted in the beginning of this section, we need to show the converse inequality. Given $\varepsilon>0$, there are normal operators $x^{\prime}, y^{\prime} \in \mathscr{M}$ with finite spectra such that $\left\|x^{\prime}-x\right\|<\varepsilon, h\left(\sigma\left(x^{\prime}\right), \sigma(x)\right)<\varepsilon,\left\|y^{\prime}-y\right\|<\varepsilon$ and $h\left(\sigma\left(y^{\prime}\right), \sigma(y)\right)<\varepsilon$. Writing $x^{\prime}=\sum_{i=1}^{m} \alpha_{i} p_{i}$ and $y^{\prime}=\sum_{j=1}^{n} \beta_{j} q_{j}$ where $\sum_{i=1}^{m} p_{i}=\sum_{j=1}^{n} q_{j}=1, p_{i} \neq 0$ and $q_{j} \neq 0$, we choose $k(1), \ldots, k(m)$ and $l(1), \ldots, l(n)$ so that

$$
\begin{aligned}
\left|\alpha_{i}-\beta_{k(i)}\right| & =\min _{1 \leq j \leq n}\left|\alpha_{i}-\beta_{j}\right|, & 1 \leq i \leq m, \\
\left|\alpha_{l(j)}-\beta_{j}\right| & =\min _{1 \leq i \leq m}\left|\alpha_{i}-\beta_{j}\right|, & 1 \leq j \leq n,
\end{aligned}
$$

and divide $p_{i}$ and $q_{j}$ into nonzero projections as follows:

$$
\begin{array}{ll}
p_{i}=p_{i}^{\prime}+\sum_{j: l(j)=i} p_{j}^{\prime \prime}, & 1 \leq i \leq m, \\
q_{j}=q_{j}^{\prime}+\sum_{i: k(i)=j} q_{i}^{\prime \prime}, & 1 \leq j \leq n .
\end{array}
$$

Then

$$
x^{\prime}=\sum_{i=1}^{m} \alpha_{i} p_{i}^{\prime}+\sum_{j=1}^{n} \alpha_{l(j)} p_{j}^{\prime \prime}, \quad y^{\prime}=\sum_{i=1}^{m} \beta_{k(i)} q_{i}^{\prime \prime}+\sum_{j=1}^{n} \beta_{j} q_{j}^{\prime} .
$$

From the assumption of $\mathscr{M}$, there exists a $u \in \mathscr{U}$ such that $p_{i}^{\prime}=u q_{i}^{\prime \prime} u^{*}$ for all $i$ and $p_{j}^{\prime \prime}=u q_{j}^{\prime} u^{*}$ for all $j$. Hence

$$
\begin{aligned}
\left\|x^{\prime}-u y^{\prime} u^{*}\right\| & =\max \left\{\max _{1 \leq i \leq m}\left|\alpha_{i}-\beta_{k(i)}\right|, \max _{1 \leq j \leq n}\left|\alpha_{l(j)}-\beta_{j}\right|\right\} \\
& =h\left(\sigma\left(x^{\prime}\right), \sigma\left(y^{\prime}\right)\right),
\end{aligned}
$$


so that

$$
\begin{aligned}
\operatorname{dist}(\mathscr{U}(x), \mathscr{U}(y)) & \leq \operatorname{dist}\left(\mathscr{U}\left(x^{\prime}\right), \mathscr{U}\left(y^{\prime}\right)\right)+2 \varepsilon \\
& \leq h(\sigma(x), \sigma(y))+4 \varepsilon .
\end{aligned}
$$

Besides $\operatorname{dist}(\mathscr{U}(x), \mathscr{U}(y))$, the anti-distance $\sup _{u \in \mathscr{U}}\left\|x-u y u^{*}\right\|$ between $\mathscr{U}(x)$ and $\mathscr{U}(y)$ is of some interest. Concerning this, it was shown in [4] that

$$
\sup _{u \in \mathscr{U}}\left\|x-u y u^{*}\right\| \leq \sqrt{2} \max \{|\alpha-\beta|: \alpha \in \sigma(x), \beta \in \sigma(y)\}
$$

for every pair of normal operators $x$ and $y$ in $\mathscr{M}=\mathbf{B}(\mathscr{H}$ ) (hence in an arbitrary von Neumann algebra $\mathscr{M}$ ). The constant $\sqrt{2}$ is best possible even for $2 \times 2$ unitary matrices.

2. $\operatorname{dist}(\mathscr{U}(x), \mathscr{U}(y))=\delta(x, y)$ for several classes. The equality $\operatorname{dist}(\mathscr{U}(x), \mathscr{U}(y))=\delta(x, y)$ is known to hold for several classes of normal matrices, while it is still open as a long-standing conjecture whether this remains true for all normal matrices. The equality for Hermitian matrices is a classical result of Weyl [45]. See [41] for Hermitian and skew-Hermitian matrices. The equality was established in [7] (also [21]) for unitary matrices and in [10] for scalar multiples of unitary matrices. Furthermore the analogous equality holds for corresponding classes of operators in case of $\mathscr{M}=\mathbf{B}(\mathscr{H})$ (see $[6,14]$ ).

The next theorem extends the above results to the general semifinite case. For a normal operator $x$ in $\mathscr{M}$, let $\sigma_{f}(x)$ be the set of all $\alpha \in \sigma(x)$ such that $\tau\left(e_{D_{r}(\alpha)}(x)\right)<\infty$ for some $r>0$, where $D_{r}(\alpha)$ is the open disk of center $\alpha$ and radius $r$. When $\mathscr{M}=\mathbf{B}(\mathscr{H}), \sigma(x) \backslash \sigma_{f}(x)$ is the essential spectrum of $x$.

THEOREM 2.1. Assume that $\mathscr{M}$ is a $\sigma$-finite semifinite factor. Then the equality

$$
\operatorname{dist}(\mathscr{U}(x), \mathscr{U}(y))=\delta(x, y)
$$

holds for every pair of normal operators $x, y \in \mathscr{M}$ satisfying one of the following conditions:

(1) $x$ and $y$ are commuting,

(2) $\sigma_{f}(x)=\varnothing$ or $\sigma_{f}(y)=\varnothing$,

(3) $\sigma(x)$ and $\sigma(y)$ are included in parallel straight lines $L_{x}$ and $L_{y} r e-$ spectively (in particular, $x$ and $y$ are selfadjoint operators plus scalars),

(4) $\sigma(x)$ and $\sigma(y)$ are included in perpendicular straight lines $L_{x}$ and $L_{y}$ respectively (in particular, $x$ is selfadjoint and $y$ is skew-adjoint), 
(5) $\sigma(x)$ and $\sigma(y)$ are included in cocentric circles $C_{x}$ and $C_{y}$ respectively (in particular, $x$ and $y$ are scalar multiples of unitaries).

Proof. It suffices by Theorem 1.4 to prove the inequality $\|x-y\| \geq$ $\delta(x, y)$. We may assume $\delta(x, y)>0$. In the following proof of this inequality, $\mathscr{M}$ may be an arbitrary semifinite von Neumann algebra.

Case (1) can be shown as [14, Proposition 2.3].

Case (2). Assume $\sigma_{f}(x)=\varnothing$. Let $0<r<\delta(x, y)$. Then there is an open set $V \subseteq \mathbf{C}$ such that either $\tau\left(e_{V}(x)\right)>\tau\left(e_{V_{r}}(y)\right)$ or $\tau\left(e_{V}(y)\right)>$ $\tau\left(e_{V_{r}}(x)\right)$. When $\tau\left(e_{V}(x)\right)>\tau\left(e_{V_{r}}(y)\right)$, taking $\gamma \in V \cap \sigma(x)$ and $\varepsilon>0$ with $D_{\varepsilon}(\gamma) \subseteq V$, we have by $\sigma_{f}(x)=\varnothing$

$$
\tau\left(e_{D_{\varepsilon}(\gamma)}(x)\right)=\infty>\tau\left(e_{V_{r}}(y)\right) \geq \tau\left(e_{D_{\varepsilon+r}(\gamma)}(y)\right) .
$$

Hence $e_{D_{\varepsilon}(\gamma)}(x) \wedge e_{D_{\varepsilon+r}(\gamma)}(y)^{\perp} \neq 0$. Taking a unit vector $\xi$ in the range of this nonzero projection, we get

$$
\|x-y\| \geq\|(y-\gamma) \xi\|-\|(x-\gamma) \xi\| \geq(\varepsilon+r)-\varepsilon=r .
$$

When $\tau\left(e_{V}(y)\right)>\tau\left(e_{V_{r}}(x)\right)$, since $V \cap \sigma(y) \neq \varnothing$ and $V_{r} \cap \sigma(x)=\varnothing$ by $\sigma_{f}(x)=\varnothing$, it follows that $\|x-y\| \geq h(\sigma(x), \sigma(y)) \geq r$. Thus $\|x-y\| \geq \delta(x, y)$.

Case (3). Multiplying $x$ and $y$ by a nonzero scalar, we may assume that $L_{x}$ and $L_{y}$ are parallel to the real line. For two points $\alpha$ and $\alpha^{\prime}$ on $L_{x}$ (or $L_{y}$ ), the open interval on $L_{x}$ (or $L_{y}$ ) with end points $\alpha$ and $\alpha^{\prime}$ is denoted by $\left(\alpha, \alpha^{\prime}\right)$. Here let $\left(\alpha, \alpha^{\prime}\right)=\varnothing$ unless $\alpha^{\prime}$ is on the right-side of $\alpha$. We first show that $\delta(x, y)$ is equal to the infimum of $r>0$ such that $\tau\left(e_{I}(x)\right) \leq \tau\left(e_{I_{r}}(y)\right)$ for every bounded open interval $I$ on $L_{x}$ and $\tau\left(e_{J}(y)\right) \leq \tau\left(e_{J_{r}}(x)\right)$ for every bounded open interval $J$ on $L_{y}$. Let $d$ be this infimum and $d_{0}$ the distance between $L_{x}$ and $L_{y}$. Then it is immediately seen that $d_{0} \leq d \leq \delta(x, y)$. So we need to check that if $r>d_{0}$ and $\tau\left(e_{I}(x)\right) \leq \tau\left(e_{I_{r}}(y)\right)$ for every bounded open interval $I$ on $L_{x}$, then $\tau\left(e_{V}(x)\right) \leq \tau\left(e_{V_{r}}(y)\right)$ for every open set $V \subseteq \mathbf{C}$. For any bounded open set $V \subseteq \mathbf{C}, V_{r} \cap L_{y}$ is the disjoint countable union of open intervals $J_{n}=\left(\beta_{n}, \beta_{n}^{\prime}\right)$. Let $\alpha_{n}$ (resp. $\left.\alpha_{n}^{\prime}\right)$ be the right-hand (resp. left-hand) point of two intersections of $L_{x}$ with $C_{r}\left(\beta_{n}\right)$ (resp. $\left.C_{r}\left(\beta_{n}^{\prime}\right)\right)$, where $C_{r}(\gamma)$ denotes the circle of center $\gamma$ and radius $r$. Define $I_{n}=\left(\alpha_{n}, \alpha_{n}^{\prime}\right)$. Then $V \cap L_{x} \subseteq \bigcup_{n} I_{n}$. In fact, for each $\gamma \in V \cap L_{x}$, let $\beta$ and $\beta^{\prime}$ be two intersections of $L_{y}$ with $C_{r}(\gamma)$. Since $\left(\beta, \beta^{\prime}\right) \subseteq V_{r} \cap L_{y},\left(\beta, \beta^{\prime}\right)$ is included in some $J_{n}$, so $\gamma \in I_{n}$. Moreover 
the $I_{n}$ are mutually disjoint and $\left(I_{n}\right)_{r} \cap L_{y} \subseteq J_{n}$ by definition. Hence

$$
\begin{aligned}
\tau\left(e_{V}(x)\right) & \leq \sum_{n} \tau\left(e_{I_{n}}(x)\right) \leq \sum_{n} \tau\left(e_{\left(I_{n}\right)_{r}}(y)\right) \\
& \leq \sum_{n} \tau\left(e_{J_{n}}(y)\right)=\tau\left(e_{V_{r}}(y)\right) .
\end{aligned}
$$

Thus $\delta(x, y)=d$ is verified.

Now suppose $\|x-y\|<\delta(x, y)$ on the contrary and let $\|x-y\|<$ $r<\delta(x, y)$. By the fact shown above, we assume without loss of generality that $\tau\left(e_{I}(x)\right)>\tau\left(e_{I_{r}}(y)\right)$ for some open interval $I=\left(\alpha, \alpha^{\prime}\right)$ on $L_{x}$. Since

$$
d_{0} \leq h(\sigma(x), \sigma(y)) \leq\|x-y\|<r,
$$

$I_{r} \cap L_{y}$ is an open interval $\left(\beta, \beta^{\prime}\right)$ and the length of $\overline{\alpha \beta}$ (also $\overline{\alpha^{\prime} \beta^{\prime}}$ ) is $r(\overline{\alpha \beta}$ denotes the line segment joining $\alpha$ and $\beta)$. When $L_{x}=L_{y}$, let $\gamma$ be the midpoint of $\overline{\alpha \alpha^{\prime}}$. Otherwise the lines $\alpha \beta$ and $\alpha^{\prime} \beta^{\prime}$ meet at some $\gamma$. Let $s$ be the length of $\overline{\gamma \alpha}$ (also $\left.\overline{\gamma \alpha^{\prime}}\right)$. Then

$$
I \subseteq D_{s}(\gamma), \quad\left(L_{y} \backslash I_{r}\right) \cap D_{s+r}(\gamma)=\varnothing .
$$

Taking a unit vector $\xi$ in the range of $e_{I}(x) \wedge e_{I_{r}}(y)^{\perp}$, we get

$$
\|x-y\| \geq\|(y-\gamma) \xi\|-\|(x-\gamma) \xi\| \geq(s+r)-s=r,
$$

a contradiction.

Case (4). Transforming $x$ and $y$ by a linear function, we may assume that $x$ is selfadjoint and $y$ is skew-adjoint. Let $d$ be the infimum of $r>0$ such that for every $0 \leq s \leq r$

(i) $\tau\left(e_{[s, \infty)}(|x|)\right) \leq \tau\left(e_{\left[0, \sqrt{r^{2}-s^{2}}\right)}(|y|)\right)$,

(ii) $\tau\left(e_{[s, \infty)}(|y|)\right) \leq \tau\left(e_{\left[0, \sqrt{r^{2}-s^{2}}\right)}(|x|)\right)$.

Given $r>0$, suppose that the above (i) holds for every $0 \leq s \leq r$. Letting $s=r$, we get $\tau\left(e_{[r, \infty)}(|x|)\right)=0$. For any open set $V \subseteq \mathbf{C}$, let $s=\inf \{|t|: t \in V \cap \mathbf{R}\}$. When $s \geq r, \tau\left(e_{V}(x)\right)=0 \leq \tau\left(e_{V_{r}}(y)\right)$. Otherwise we have

$$
\begin{aligned}
\tau\left(e_{V}(x)\right) & \leq \tau\left(e_{[s, \infty)}(|x|)\right) \\
& \leq \tau\left(e_{\left[0, \sqrt{r^{2}-s^{2}}\right)}(|y|)\right) \leq \tau\left(e_{V_{r}}(y)\right)
\end{aligned}
$$

since $i\left(-\sqrt{r^{2}-s^{2}}, \sqrt{r^{2}-s^{2}}\right) \subseteq V_{r} \cap i \mathbf{R}$. Conversely suppose that $\tau\left(e_{V}(x)\right) \leq \tau\left(e_{V_{r}}(y)\right)$ for every open set $V \subseteq \mathbf{C}$. For each $t>r$ and $0 \leq s \leq t$, there is an open set $V \subseteq \mathbf{C}$ such that

$$
\begin{aligned}
V & \cap \mathbf{R} \supseteq(-\infty,-s] \cup[s, \infty), \\
V_{r} & \cap i \mathbf{R} \subseteq i\left(-\sqrt{t^{2}-s^{2}}, \sqrt{t^{2}-s^{2}}\right),
\end{aligned}
$$


and hence

$$
\begin{aligned}
\tau\left(e_{[s, \infty)}(|x|)\right) & \leq \tau\left(e_{V}(x)\right) \\
& \leq \tau\left(e_{V_{r}}(y)\right) \leq \tau\left(e_{\left[0, \sqrt{t^{2}-s^{2}}\right)}(|y|)\right) .
\end{aligned}
$$

Together with the same argument where $x$ and $y$ are exchanged, we obtain $\delta(x, y)=d$.

Now let $0<r<\delta(x, y)$. Then there exists an $s \in[0, r]$ for which either (i) or (ii) above is violated. If (i) is false, then a unit vector $\xi$ can be chosen in the range of $e_{[s, \infty)}(|x|) \wedge e_{\left[\sqrt{r^{2}-s^{2}}, \infty\right)}(|y|)$. So, as in [41], we have by $(x-y)^{*}=x+y$

$$
\begin{aligned}
\|x-y\|^{2} & =\frac{1}{2}\left\{\|x-y\|^{2}+\|x+y\|^{2}\right\} \\
& \geq \frac{1}{2}\left\{\|(x-y) \xi\|^{2}+\|(x+y) \xi\|^{2}\right\} \\
& =\|x \xi\|^{2}+\|y \xi\|^{2} \geq s^{2}+\left(r^{2}-s^{2}\right)=r^{2},
\end{aligned}
$$

implying $\|x-y\| \geq \delta(x, y)$.

Case (5). This will be proved in a manner analogous to the case (3). Moreover it should be noted that the idea of proof is essentially the same as that used in [21] for unitary matrices. For $\alpha, \alpha^{\prime} \in C_{x}$ (or $C_{y}$ ), the open arc joining $\alpha$ and $\alpha^{\prime}$ counter-clockwise on $C_{x}$ (or $\left.C_{y}\right)$ is denoted by $\left(\alpha, \alpha^{\prime}\right)$. Let $d$ be the infimum of $r>0$ such that $\tau\left(e_{I}(x)\right) \leq \tau\left(e_{I_{r}}(y)\right)$ for every open $\operatorname{arc} I$ on $C_{x}$ and $\tau\left(e_{J}(y)\right) \leq \tau\left(e_{J_{r}}(x)\right)$ for every open arc $J$ on $C_{y}$. Also let $d_{0}=\left|r_{x}-r_{y}\right|$ and $d_{1}=r_{x}+r_{y}$ where $r_{x}$ and $r_{y}$ are the radii of $C_{x}$ and $C_{y}$, respectively. Then it is immediate that $d_{0} \leq d \leq \delta(x, y) \leq d_{1}$. To show $\delta(x, y)=d$, it suffices to check that if $d_{0}<r<d_{1}$ and $\tau\left(e_{I}(x)\right) \leq \tau\left(e_{I_{r}}(y)\right)$ for every open $\operatorname{arc} I$ on $C_{x}$, then $\tau\left(e_{V}(x)\right) \leq \tau\left(e_{V_{r}}(y)\right)$ for every open set $V \subseteq \mathbf{C}$. For any open set $V \subseteq \mathbf{C}, V_{r} \cap C_{y}$ is the disjoint countable union of open $\operatorname{arcs} J_{n}=\left(\beta_{n}, \beta_{n}^{\prime}\right)$. Let $\alpha_{n}$ (resp. $\alpha_{n}^{\prime}$ ) be the end point (resp. start point) of the arc on $C_{x}$ which joins two intersections of $C_{x}$ with $C_{r}\left(\beta_{n}\right)$ (resp. $\left.C_{r}\left(\beta_{n}^{\prime}\right)\right)$ and lies on the side near $\beta_{n}$ (resp. $\left.\beta_{n}^{\prime}\right)$. Define $I_{n}=\left(\alpha_{n}, \alpha_{n}^{\prime}\right)$ if the segments $\overline{\alpha_{n} \beta_{n}}$ and $\overline{\alpha_{n}^{\prime} \beta_{n}^{\prime}}$ do not intersect, and $I_{n}=\varnothing$ if they do. Then, as in case (3), $V \cap C_{x} \subseteq \bigcup_{n} I_{n}$ (disjoint union) and $\left(I_{n}\right)_{r} \cap C_{y} \subseteq J_{n}$, so that we get $\tau\left(e_{V}(x)\right) \leq \tau\left(e_{V_{r}}(y)\right)$. Thus $\delta(x, y)=d$.

Now suppose that $\|x-y\|<r<\delta(x, y)$ and so $\tau\left(e_{I}(x)\right)>\tau\left(e_{I_{r}}(y)\right)$ for some open arc $I=\left(\alpha, \alpha^{\prime}\right)$ on $C_{x}$. Since

$$
d_{0} \leq h(\sigma(x), \sigma(y)) \leq\|x-y\|<r,
$$


$I_{r} \cap C_{y}$ is an open $\operatorname{arc}\left(\beta, \beta^{\prime}\right)$ and the length of $\overline{\alpha \beta}$ (also $\overline{\alpha^{\prime} \beta^{\prime}}$ ) is $r$. Assume that the lines $\alpha \beta$ and $\alpha^{\prime} \beta^{\prime}$ meet at some $\gamma$, and let $s$ be the shorter length of $\overline{\gamma \alpha}$ and $\overline{\gamma \beta}$. Then either

$$
I \subseteq D_{s}(\gamma), \quad\left(C_{y} \backslash I_{r}\right) \cap D_{s+r}(\gamma)=\varnothing,
$$

or

$$
I \cap D_{s+r}(\gamma)=\varnothing, \quad C_{y} \backslash I_{r} \subseteq \overline{D_{s}(\gamma)} .
$$

In either cases, as in (3) we get $\|x-y\| \geq r$, a contradiction. When the lines $\alpha \beta$ and $\alpha^{\prime} \beta^{\prime}$ are parallel, we have a contradiction as well by taking a point $\gamma$ far enough on the line $\alpha \beta$.

In the above proof, various new expressions of $\delta(x, y)$ have been given for cases (3)-(5) of Theorem 2.1. Those may be useful in the computation of $\operatorname{dist}(\mathscr{U}(x), \mathscr{U}(y))$.

3. Submajorizations for spectral scales. A densely defined closed operator $x$ affiliated with $\mathscr{M}$ is said to be $\tau$-measurable if there is, for each $\varepsilon>0$, a projection $e$ in $\mathscr{M}$ such that $e \mathscr{H} \subseteq \mathscr{D}(x)$ and $\tau\left(e^{\perp}\right)<\varepsilon$. We denote by $\tilde{\mathscr{M}}$ the set of all $\tau$-measurable operators affiliated with $\mathscr{M}$, which becomes a complete Hausdorff topological *-algebra in the measure topology (see $[34,42])$. For each $x \in \tilde{\mathscr{M}}$ and $0<p \leq \infty$, the $L^{p}$-(quasi-)norm $\|x\|_{p}(\in[0, \infty])$ of $x$ is defined by $\|x\|_{p}=\tau\left(|x|^{p}\right)^{1 / p}$ when $0<p<\infty$ and $\|x\|_{\infty}=\|x\|$. Then the noncommutative $L^{p}$-space $L^{p}(\mathscr{M})=L^{p}(\mathscr{M} ; \tau)$ on $(\mathscr{M}, \tau)$ is given by $L^{p}(\mathscr{M})=\left\{x \in \tilde{\mathscr{M}}:\|x\|_{p}<\infty\right\}$. When $1 \leq p \leq \infty, L^{p}(\mathscr{M})$ is a Banach space with the norm $\|\cdot\|_{p}$ (see $\left.[15,34,39,46]\right)$. Moreover we denote by $\tilde{\mathfrak{S}}$ the set of all $x \in \tilde{\mathscr{M}}$ such that $\tau\left(e_{(s, \infty)}(|x|)\right)<\infty$ for every $s>0$. Then $\tilde{\mathfrak{S}}$ is the closure of $L^{p}(\mathscr{M})$ in the measure topology where $0<p<\infty$. If $\tau(1)<\infty$, then $\tilde{\mathfrak{S}}=\tilde{\mathscr{M}}$ which is the set of all densely defined closed operators affiliated with $\mathscr{M}$. In particular when $\mathscr{M}=\mathbf{B}(\mathscr{H}), L^{p}(\mathscr{M})$ is the Schatten-von Neumann $p$-class and $\tilde{\mathfrak{S}}$ is the algebra of all compact operators on $\mathscr{H}$. For each subspace $\mathscr{L}$ of $\tilde{\mathscr{M}}$, the set of all selfadjoint (resp. positive selfadjoint) operators in $\mathscr{L}$ is denoted by $\mathscr{L}_{s a}\left(\right.$ resp. $\left.\mathscr{L}_{+}\right)$.

For each $x \in \tilde{\mathscr{M}}$ and $t>0$, the generalized s-number $\mu_{t}(x)$ is defined by

$$
\mu_{t}(x)=\inf \left\{s \geq 0: \tau\left(e_{(s, \infty)}(|x|)\right) \leq t\right\} .
$$

Denote simply by $\mu(x)$ the function $t \mapsto \mu_{t}(x)$ on $(0, \infty)$ into $[0, \infty)$. A detailed exposition on generalized $s$-numbers is found in [17] (also $[35,46])$. When $\tau(1)<\infty$, for $x \in \tilde{\mathscr{M}}_{s a}$ we define

$$
\lambda_{t}(x)=\inf \left\{s \in \mathbf{R}: \tau\left(e_{(s, \infty)}(x)\right) \leq t\right\}, \quad 0<t<\tau(1),
$$


and call it the spectral scale of $x$ following [36]. Furthermore define $\check{\lambda}_{t}(x)=-\lambda_{t}(-x)$, i.e. $\check{\lambda}_{t}(x)=\lambda_{\tau(1)-t-0}(x)$ for $0<t<\tau(1)$. The function $t \mapsto \lambda_{t}(x)$ (resp. $t \mapsto \check{\lambda}_{t}(x)$ ) on $(0, \tau(1))$ into $\mathbf{R}$ is denoted by $\lambda(x)$ (resp. $\check{\lambda}(x)$ ) which is non-increasing (resp. non-decreasing) and right-continuous. Even when $\tau(1)$ is not necessarily finite, for $x \in \tilde{\mathscr{M}}_{s a}$ with the Jordan decomposition $x=x_{+}-x_{-}$, we define the functions $\boldsymbol{\lambda}(x)$ and $\check{\boldsymbol{\lambda}}(x)$ on $\mathbf{R}$ into $\mathbf{R}$ as follows:

$$
\begin{array}{r}
\boldsymbol{\lambda}_{t}(x)= \begin{cases}\mu_{t}\left(x_{+}\right), & t>0, \\
0, & t=0, \\
-\mu_{-t}\left(x_{-}\right), & t<0,\end{cases} \\
\check{\boldsymbol{\lambda}}_{t}(x)=-\boldsymbol{\lambda}_{t}(-x)= \begin{cases}-\mu_{t}\left(x_{-}\right), & t>0, \\
0, & t=0, \\
\mu_{-t}\left(x_{+}\right), & t<0 .\end{cases}
\end{array}
$$

An interval of $\mathbf{R}$ is considered as the measure space with Lebesgue measure. For $0<p \leq \infty$, we have $\|x\|_{p}=\|\mu(x)\|_{p}$ for all $x \in \tilde{\mathscr{M}}$ and $\|x\|_{p}=\|\lambda(x)\|_{p}\left(=\|\lambda(x)\|_{p}\right.$ if $\left.\tau(1)<\infty\right)$ for all $x \in \tilde{\mathscr{M}}_{s a}$ (see $\left.[\mathbf{1 7}, \mathbf{2 4}]\right)$.

In particular, let $\mathscr{M}$ be commutative, that is, $\mathscr{M}=L^{\infty}(\Omega)$ and $\tau(f)=\int_{\Omega} f d m$ on a localizable measure space $(\Omega, m)$. Then $\tilde{\mathscr{M}}$ consists of all measurable functions on $\Omega$ bounded except on $m$-finite sets. For a real measurable function $f$ on $\Omega$, the decreasing rearrangement $f^{*}$ of $f$ is given by

$$
f^{*}(t)=\inf \{s \in \mathbf{R}: m(\{\omega \in \Omega: f(\omega)>s\}) \leq t\}, \quad 0<t<m(\Omega) .
$$

Then $\mu_{t}(f)=|f|^{*}(t)$ for every $f \in \tilde{\mathscr{M}}$ and $0<t<m(\Omega)$. When $m(\Omega)<\infty, \lambda(f)=f^{*}$ for every real measurable function $f$ on $\Omega$. In this section, we shall discuss (sub)majorizations of functions relevant to the spectral scales of $\tau$-measurable selfadjoint operators. So we define the notions of majorization and submajorization in the commutative case (see [22-24] for the formulation and characterizations of (sub)majorization in the noncommutative case). For nonnegative measurable functions $f$ and $g$ on $\Omega, f$ is said to be submajorized by $g$, in notation $f \prec g$, if $\int_{0}^{s} f^{*}(t) d t \leq \int_{0}^{s} g^{*}(t) d t$ for all $s \in(0, m(\Omega))$. Furthermore, for real $f, g \in L^{1}(\Omega)$ where $m(\Omega)<\infty, f$ is said to be majorized by $g$, in notation $f \prec g$, if $\int_{0}^{s} f^{*}(t) d t \leq \int_{0}^{s} g^{*}(t) d t$ for all $x \in(0, m(\Omega))$ and $\int_{0}^{m(\Omega)} f^{*}(t) d t=\int_{0}^{m(\Omega)} g^{*}(t) d t$ (i.e. $\int_{\Omega} f d m=$ $\left.\int_{\Omega} g d m\right)$. In the following discussions, $(0, \tau(1))$ or $\mathbf{R}$ will be taken as $\Omega$. 
Using the real interpolation method, we showed in [24] that if $\tau(1)<$ $\infty$ and $x, y \in L^{1}(\mathscr{M})_{s a}$, then

$$
\lambda(x)-\lambda(y) \prec \lambda(x-y) \prec \lambda(x)-\grave{\lambda}(y) .
$$

This is the extension of the Lidskii-Wielandt theorem on the eigenvalues of Hermitian matrices. By virtue of the above majorizations, we shall establish the next theorem.

THEOREM 3.1. (1) If $\tau(1)<\infty$ and $x, y \in \tilde{\mathscr{M}}_{s a}$, then

$$
|\lambda(x)-\lambda(y)| \prec|\lambda(x-y)| \prec|\lambda(x)-\check{\lambda}(y)| \text {. }
$$

(2) If $\tau(1)=\infty$ and $x, y \in \tilde{\mathfrak{S}}_{s a}$, then

$$
|\lambda(x)-\lambda(y)| \prec|\lambda(x-y)| \prec|\lambda(x)-\check{\lambda}(y)| \text {. }
$$

We first give the following elementary lemma.

LEMMA 3.2. (1) If $\tau(1)<\infty$ and $\left\{x_{n}\right\}$ is a sequence in $\tilde{\mathscr{M}}_{s a}$ converging to $x \in \tilde{\mathscr{M}}_{s a}$ in the measure topology, then $\lambda_{t}(x)=\lim _{n \rightarrow \infty} \lambda_{t}\left(x_{n}\right)$ for every $t \in(0, \tau(1))$ at which $\lambda(x)$ is continuous.

(2) For every $x \in \tilde{\mathscr{M}}_{s a}, \mu(x)=|\lambda(x)|^{*}\left(=|\lambda(x)|^{*}\right.$ if $\left.\tau(1)<\infty\right)$.

Proof. (1) It follows as [17, Lemma 2.5(v)] that if $y, z \in \tilde{\mathscr{M}}_{s a}$ and $s, t, s+t \in(0, \tau(1))$, then

$$
\lambda_{s+t}(y+z) \leq \lambda_{s}(y)+\lambda_{t}(z) .
$$

Also $\lambda_{t}(y) \leq \mu_{t}(y)$ for every $y \in \tilde{\mathscr{M}}_{s a}$ and $t \in(0, \tau(1))$. Hence, for each $t \in(0, \tau(1))$ and $\varepsilon>0$ with $t \pm \varepsilon \in(0, \tau(1))$, we have

$$
\begin{aligned}
\lambda_{t+\varepsilon}(x) & \leq \lambda_{t}\left(x_{n}\right)+\mu_{\varepsilon}\left(x-x_{n}\right), \\
\lambda_{t}\left(x_{n}\right) & \leq \lambda_{t-\varepsilon}(x)+\mu_{\varepsilon}\left(x-x_{n}\right) .
\end{aligned}
$$

Since $\lim _{n \rightarrow \infty} \mu_{\varepsilon}\left(x-x_{n}\right)=0$ (see [17, Lemma 3.1]), we get the assertion letting $n \rightarrow \infty$ and then $\varepsilon \rightarrow 0$ in the above.

(2) Denoting Lebesgue measure by $m$, we have for $s \geq 0$

$$
\begin{aligned}
m(\{t & \left.\left.\in \mathbf{R}:\left|\lambda_{t}(x)\right|>s\right\}\right) \\
& =m\left(\left\{t>0: \mu_{t}\left(x_{+}\right)>s\right\}\right)+m\left(\left\{t>0: \mu_{t}\left(x_{-}\right)>s\right\}\right) \\
& =\tau\left(e_{(s, \infty)}(|x|)\right),
\end{aligned}
$$

implying $\mu(x)=|\lambda(x)|^{*}$. When $\tau(1)<\infty$, also $\mu(x)=|\lambda(x)|^{*}$ is easily verified. 
Proof of Theorem 3.1. (1) If $x, y \in L^{1}(\mathscr{M})_{s a}$, then the desired submajorizations follow from the majorizations mentioned before Theorem 3.1 and [11, Corollary 2.6]. Let $x, y \in \tilde{\mathscr{M}}_{s a}$. We prove the first submajorization. Unless $\lambda(x-y) \in L^{1}(0, \tau(1))$ or equivalently unless $x-y \in L^{1}(\mathscr{M})$, then there is nothing to do, because

$$
\int_{0}^{s}|\lambda(x-y)|^{*}(t) d t=\infty \text { for all } s>0
$$

(see [38, Lemma 2.2]). So assume $x-y \in L^{1}(\mathscr{M})$ and choose a sequence $\left\{x_{n}\right\}$ in $L^{1}(\mathscr{M})_{s a}$ converging to $x$ in the measure topology. Letting $y_{n}=x_{n}-x+y$, we have $\left\{y_{n}\right\}$ in $L^{1}(\mathscr{M})_{s a}$ converging to $y$ in the measure topology. Using Lemma 3.1(1) twice, we get $\lambda_{t}\left(x_{n}\right)-\lambda_{t}\left(y_{n}\right) \rightarrow \lambda_{t}(x)-\lambda_{t}(y)$ and hence $\left|\lambda\left(x_{n}\right)-\lambda\left(y_{n}\right)\right|^{*}(t) \rightarrow$ $|\lambda(x)-\lambda(y)|^{*}(t)$ for almost every $t \in(0, \tau(1))$. Since $\left|\lambda\left(x_{n}\right)-\lambda\left(y_{n}\right)\right| \prec$ $|\lambda(x-y)|$ for all $n$, the desired submajorization follows from Fatou's lemma.

To show the second submajorization, assume $\lambda(x)-\check{\lambda}(y) \epsilon$ $L^{1}(0, \tau(1))$. Then both $\lambda(x)$ and $\check{\lambda}(y)$ are in $L^{1}(0, \tau(1))$, because $\lambda(x)$ is non-increasing while $\check{\lambda}(y)$ is non-decreasing. Hence $x, y \in L^{1}(\mathscr{M})$, so the conclusion is already verified.

(2) For $n \geq 1$, let $x_{n}=x e_{(1 / n, \infty)}(|x|)$ and $y_{n}=y e_{(1 / n, \infty)}(|y|)$. Note that $\left\|\mu\left(z_{1}\right)-\mu\left(z_{2}\right)\right\|_{\infty} \leq\left\|z_{1}-z_{2}\right\|_{\infty}$ for all $z_{1}, z_{2} \in \tilde{\mathscr{M}}$ (see the proof of [17, Proposition 2.7]). By Lemma 3.2(2), we have

$$
\begin{aligned}
& \left\|\left|\lambda\left(x_{n}-y_{n}\right)\right|^{*}-|\lambda(x-y)|^{*}\right\|_{\infty} \\
& \quad=\left\|\mu\left(x_{n}-y_{n}\right)-\mu(x-y)\right\|_{\infty} \leq\left\|\left(x_{n}-y_{n}\right)-(x-y)\right\|_{\infty} \leq 2 / n,
\end{aligned}
$$

since $\left\|x_{n}-x\right\|_{\infty} \leq 1 / n$ and $\left\|y_{n}-y\right\|_{\infty} \leq 1 / n$. Also

$$
\begin{aligned}
& \left\|\boldsymbol{\lambda}\left(x_{n}\right)-\left.\boldsymbol{\lambda}\left(y_{n}\right)\right|^{*}-|\boldsymbol{\lambda}(x)-\boldsymbol{\lambda}(y)|^{*}\right\|_{\infty} \\
& \quad \leq\left\|\left(\boldsymbol{\lambda}\left(x_{n}\right)-\boldsymbol{\lambda}\left(y_{n}\right)\right)-(\boldsymbol{\lambda}(x)-\boldsymbol{\lambda}(y))\right\|_{\infty} \leq 2 / n,
\end{aligned}
$$

since $\left\|\boldsymbol{\lambda}\left(x_{n}\right)-\lambda(x)\right\|_{\infty} \leq 1 / n$ and $\left\|\boldsymbol{\lambda}\left(y_{n}\right)-\lambda(y)\right\|_{\infty} \leq 1 / n$. Similarly

$$
\left\|\boldsymbol{\lambda}\left(x_{n}\right)-\left.\check{\lambda}\left(y_{n}\right)\right|^{*}-|\lambda(x)-\check{\lambda}(y)|^{*}\right\|_{\infty} \leq 2 / n \text {. }
$$

Because $\tau\left(e_{(1 / n, \infty)}(|x|)\right)<\infty$ and $\tau\left(e_{(1 / n, \infty)}(|y|)\right)<\infty$ from $x, y \in \tilde{\mathfrak{S}}$, we can choose a projection $e$ (depending on $n$ ) in $\mathscr{M}$ so that

$$
\begin{gathered}
e_{(1 / n, \infty)}(|x|) \vee e_{(1 / n, \infty)}(|y|) \leq e, \\
2 \tau\left(e_{(1 / n, \infty)}(|x|) \vee e_{(1 / n, \infty)}(|y|)\right)<\tau(e)<\infty .
\end{gathered}
$$

Since $x_{n}, y_{n} \in\left(\mathscr{M}_{e}\right)_{s a}$, the assertion (1) implies that

$$
\left|\lambda\left(x_{n}\right)-\lambda\left(y_{n}\right)\right| \prec\left|\lambda\left(x_{n}-y_{n}\right)\right| \prec\left|\lambda\left(x_{n}\right)-\check{\lambda}\left(y_{n}\right)\right|
$$


where $\lambda\left(x_{n}\right)$ and others are defined on $(0, \tau(e))$. By Lemma 3.2(2),

$$
\begin{aligned}
\left|\lambda\left(x_{n}-y_{n}\right)\right|^{*}(t) & =\mu_{t}\left(x_{n}-y_{n}\right) \\
& = \begin{cases}\left|\lambda\left(x_{n}-y_{n}\right)\right|^{*}(t), & 0<t<\tau(e), \\
0, & t \geq \tau(e) .\end{cases}
\end{aligned}
$$

Furthermore, noting that $\lambda_{t}\left(x_{n}\right)=\lambda_{t}\left(y_{n}\right)=0$ at $t=\tau(e) / 2$, we have

$$
\begin{aligned}
& \boldsymbol{\lambda}_{t}\left(x_{n}\right)-\boldsymbol{\lambda}_{t}\left(y_{n}\right)= \begin{cases}\lambda_{t}\left(x_{n}\right)-\lambda_{t}\left(y_{n}\right), & 0<t<\tau(e) / 2, \\
\lambda_{\tau(e)-t-0}\left(x_{n}\right)-\lambda_{\tau(e)-t-0}\left(y_{n}\right), & -\tau(e) / 2<t<0, \\
0, & \text { otherwise, }\end{cases} \\
& \boldsymbol{\lambda}_{t}\left(x_{n}\right)-\check{\boldsymbol{\lambda}}_{t}\left(y_{n}\right)= \begin{cases}\lambda_{t}\left(x_{n}\right)-\check{\lambda}_{t}\left(y_{n}\right), & 0<t<\tau(e) / 2, \\
\lambda_{\tau(e)-t-0}\left(x_{n}\right)-\check{\lambda}_{\tau(e)-t-0}\left(y_{n}\right), & -\tau(e) / 2<t<0, \\
0, & \text { otherwise, }\end{cases}
\end{aligned}
$$

so that

$$
\begin{aligned}
& \left|\lambda\left(x_{n}\right)-\lambda\left(y_{n}\right)\right|^{*}(t)= \begin{cases}\left|\lambda\left(x_{n}\right)-\lambda\left(y_{n}\right)\right|^{*}(t), & 0<t<\tau(e), \\
0, & t \geq \tau(e),\end{cases} \\
& \left|\lambda\left(x_{n}\right)-\check{\lambda}\left(y_{n}\right)\right|^{*}(t)= \begin{cases}\left|\lambda\left(x_{n}\right)-\check{\lambda}\left(y_{n}\right)\right|^{*}(t), & 0<t<\tau(e), \\
0, & t \geq \tau(e) .\end{cases}
\end{aligned}
$$

These all together imply that

$$
\left|\lambda\left(x_{n}\right)-\lambda\left(y_{n}\right)\right| \prec\left|\lambda\left(x_{n}-y_{n}\right)\right| \prec\left|\lambda\left(x_{n}\right)-\check{\lambda}\left(y_{n}\right)\right|
$$

for all $n$. Therefore we get the desired conclusion by passing to the limit as $n \rightarrow \infty$.

Remark 3.3. (1) In view of Lemma 3.2(2) and [40, p. 202], the first submajorizations in (1) and (2) of Theorem 3.1 are described as follows: for each Borel subset $E$ of $(0, \tau(1))$,

$$
\int_{E}\left|\lambda_{t}(x)-\lambda_{t}(y)\right| d t \leq \int_{0}^{m(E)} \mu_{t}(x-y) d t
$$

and for each Borel subset $E$ and $F$ of $(0, \infty)$,

$$
\begin{gathered}
\int_{E}\left|\mu_{t}\left(x_{+}\right)-\mu_{t}\left(y_{+}\right)\right| d t+\int_{F}\left|\mu_{t}\left(x_{-}\right)-\mu_{t}\left(y_{-}\right)\right| d t \\
\leq \int_{0}^{m(E)+m(F)} \mu_{t}(x-y) d t .
\end{gathered}
$$

An analogous result was earlier given in [31, Theorem 5.1] for selfadjoint compact operators in case of $\mathscr{M}=\mathbf{B}(\mathscr{H})$. 
(2) In [24], besides the majorizations before Theorem 3.1, we established $|\mu(x)-\mu(y)| \prec \mu(x-y)$ for every $x \in \tilde{\mathscr{M}}$ and $y \in \tilde{\mathfrak{S}}$. Restricted to $x, y \in \tilde{\mathfrak{S}}_{s a}$ with $\tau(1)=\infty$, the first submajorization in Theorem 3.1(2) improves the above because

$$
\begin{aligned}
|\mu(x)-\mu(y)| & =|\boldsymbol{\lambda}(x)|^{*}-|\boldsymbol{\lambda}(y)|^{*} \mid \\
& \prec|\boldsymbol{\lambda}(x)-\boldsymbol{\lambda}(y)|^{*} \prec|\boldsymbol{\lambda}(x-y)|^{*}=\mu(x-y) .
\end{aligned}
$$

4. $L^{p}$-distance between unitary orbits of selfadjoint operators. In this section, we shall exactly estimate the $L^{p}$-distance $\inf _{u \in \mathscr{U}}\left\|x-u y u^{*}\right\|_{p}$ and the anti- $L^{p}$-distance $\sup _{u \in \mathscr{U}}\left\|x-u y u^{*}\right\|_{p}$ for $\tau$-measurable selfadjoint operators $x$ and $y$ in terms of their spectral scales.

To state and prove our theorems on $L^{p}$-distances, we here introduce the notion of spectral equivalence between operators in $\tilde{\mathfrak{S}}_{s a}$. Given $x, y \in \tilde{\mathfrak{S}}_{s a}$, we say that $x$ is spectrally equivalent to $y$, in notation $x \approx y$, if $\boldsymbol{\lambda}_{t}(x)=\boldsymbol{\lambda}_{t}(y)$ for all $t \in \mathbf{R}$. When $\tau(1)<\infty$, this is equivalent to $\lambda_{t}(x)=\lambda_{t}(y)$ for all $t \in(0, \tau(1))$.

LEMma 4.1. Assume that $\mathscr{M}$ is a semifinite factor and $x, y \in \tilde{\mathfrak{S}}_{s a}$. If $x \approx y$, then there exists a sequence $\left\{u_{n}\right\}$ in $\mathscr{U}$ such that $\left\|x-u_{n} y u_{n}^{*}\right\|_{p} \rightarrow$ 0 for all $1 \leq p \leq \infty$.

Proof. For a countable partition $\Delta: 0<\cdots<t_{-2}<t_{-1}<t_{0}<t_{1}<$ $t_{2}<\cdots<\infty$ of $(0, \infty)$ where $t_{-n} \rightarrow 0$ and $t_{n} \rightarrow \infty$ as $n \rightarrow \infty$, define

$$
\begin{aligned}
& x_{\Delta}=\sum_{i=-\infty}^{\infty} t_{i} e_{\left(t_{t-1}, t_{i}\right]}\left(x_{+}\right)-\sum_{i=-\infty}^{\infty} t_{i} e_{\left(t_{t-1}, t_{t}\right]}\left(x_{-}\right), \\
& y_{\Delta}=\sum_{i=-\infty}^{\infty} t_{i} e_{\left(t_{t-1}, t_{t}\right]}\left(y_{+}\right)-\sum_{i=-\infty}^{\infty} t_{i} e_{\left(t_{t-1}, t_{t}\right]}\left(y_{-}\right) .
\end{aligned}
$$

From $x, y \in \tilde{\mathfrak{S}}_{s a}$, it is easy to see that, for each $\varepsilon>0$, there exists a partition $\Delta$ as above for which $\left\|x_{\Delta}-x\right\|_{p}<\varepsilon$ and $\left\|y_{\Delta}-y\right\|_{p}<\varepsilon$ hold for $p=1, \infty$. Let

$$
\begin{array}{rlrl}
p_{i} & =e_{\left(t_{t-1}, t_{i}\right]}\left(x_{+}\right), & & p_{i}^{-}=e_{\left(t_{t-1}, t_{l}\right]}\left(x_{-}\right), \\
q_{i}=e_{\left(t_{t-1}, t_{i}\right]}\left(y_{+}\right), & q_{i}^{-}=e_{\left(t_{t-1}, t_{i}\right]}\left(y_{-}\right) .
\end{array}
$$

Since $x, y \in \tilde{\mathfrak{S}}_{s a}$ and $x \approx y$, we have $\tau\left(p_{i}\right)=\tau\left(q_{i}\right)<\infty$ and $\tau\left(p_{i}^{-}\right)=$ $\tau\left(q_{i}^{-}\right)<\infty$ for all $i$. Therefore, since $\mathscr{M}$ is a factor, $p_{i} \sim q_{i}$ and $p_{i}^{-} \sim q_{i}^{-}$in the Murray-von Neumann sense for all $i$. First assume that $\tau\left(e_{(0, \infty)}(|x|)\right)<\infty$. Then $e_{\{0\}}(x) \sim e_{\{0\}}(y)$ as well. So there exists 
a $u \in \mathscr{U}$ such that $e_{\{0\}}(x)=u e_{\{0\}}(y) u^{*}, p_{i}=u q_{i} u^{*}$ and $p_{i}^{-}=u q_{i}^{-} u^{*}$ for all $i$. Hence $x_{\Delta}=u y_{\Delta} u^{*}$, implying

$$
\left\|x-u y u^{*}\right\|_{p} \leq\left\|x-x_{\Delta}\right\|_{p}+\left\|y_{\Delta}-y\right\|_{p}<2 \varepsilon, \quad p=1, \infty .
$$

Next assume that $\tau\left(e_{(0, \infty)}(|x|)\right)=\infty$. We may assume without loss of generality that $\sum_{i=-\infty}^{-1} \tau\left(p_{i}\right)=\infty$. Choose integers $0>n_{1} \geq m_{1}>$ $n_{2} \geq m_{2}>\cdots$ such that $t_{n_{k}}<\varepsilon / 2^{k}$ and

$$
\sum_{i=m_{k}+1}^{n_{k}} \tau\left(p_{i}\right)<1 \leq \sum_{i=m_{k}}^{n_{k}} \tau\left(p_{i}\right), \quad k \geq 1 .
$$

Now we take projections $p_{i}^{\prime}$ and $q_{i}^{\prime}$ in $\mathscr{M}$ for $i=-1,-2, \ldots$ as follows. Let $p_{i}^{\prime}=q_{i}^{\prime}=0$ for $n_{1}<i<0$ and $n_{k+1}<i<m_{k}, k \geq 1$. Let $p_{i}^{\prime}=p_{i}$ and $q_{i}^{\prime}=q_{i}$ for $m_{k}<i \leq n_{k}, k \geq 1$. Then $p_{m_{k}}^{\prime} \leq p_{m_{k}}$ and $q_{m_{k}}^{\prime} \leq q_{m_{k}}$ can be taken so that

$$
\sum_{i=m_{k}}^{n_{k}} \tau\left(p_{i}^{\prime}\right)=\sum_{i=m_{k}}^{n_{k}} \tau\left(q_{i}^{\prime}\right)=1, \quad k \geq 1 .
$$

This construction gives $\tau\left(p_{i}^{\prime}\right)=\tau\left(q_{i}^{\prime}\right), \tau\left(\sum_{i=-\infty}^{-1} p_{i}^{\prime}\right)=\tau\left(\sum_{i=-\infty}^{-1} q_{i}^{\prime}\right)=$ $\infty,\left\|\sum_{i=-\infty}^{-1} t_{i} p_{i}^{\prime}\right\|_{p}<\varepsilon$ and $\left\|\sum_{i=-\infty}^{-1} t_{i} q_{i}^{\prime}\right\|_{p}<\varepsilon$ for $p=1, \infty$. Here note that the above construction is valid in the type I case (i.e. $\mathscr{M}=\mathbf{B}(\mathscr{H})$ with the canonical trace) as well as in the type II case. Defining

$$
\begin{aligned}
& x_{\Delta}^{\prime}=x_{\Delta}-\sum_{i=-\infty}^{-1} t_{i} p_{i}^{\prime}=\sum_{i=-\infty}^{-1} t_{i}\left(p_{i}-p_{i}^{\prime}\right)+\sum_{i=0}^{\infty} t_{i} p_{i}-\sum_{i=-\infty}^{\infty} t_{i} p_{i}^{-}, \\
& y_{\Delta}^{\prime}=y_{\Delta}-\sum_{i=-\infty}^{-1} t_{i} q_{i}^{\prime}=\sum_{i=-\infty}^{-1} t_{i}\left(q_{i}-q_{i}^{\prime}\right)+\sum_{i=0}^{\infty} t_{i} q_{i}-\sum_{i=-\infty}^{\infty} t_{i} q_{i}^{-},
\end{aligned}
$$

we get $\left\|x_{\Delta}^{\prime}-x\right\|_{p}<2 \varepsilon$ and $\left\|y_{\Delta}^{\prime}-y\right\|_{p}<2 \varepsilon$ for $p=1, \infty$. Because $p_{i}-p_{i}^{\prime} \sim q_{i}-q_{i}^{\prime}$ for $i=-1,-2, \ldots$ and

$$
e_{\{0\}}(x)+\sum_{i=-\infty}^{-1} p_{i}^{\prime} \sim e_{\{0\}}(y)+\sum_{i=-\infty}^{-1} q_{i}^{\prime},
$$

there exists a $u \in \mathscr{U}$ such that $x_{\Delta}^{\prime}=u y_{\Delta}^{\prime} u^{*}$ and hence $\left\|x-u y u^{*}\right\|_{p}<$ $4 \varepsilon, p=1, \infty$. The above assertion shows that there is a sequence $\left\{u_{n}\right\}$ in $\mathscr{U}$ such that $\left\|x-u_{n} y u_{n}^{*}\right\|_{p} \rightarrow 0, p=1, \infty$. Since

$$
\left\|x-u_{n} y u_{n}^{*}\right\|_{p} \leq\left\|x-u_{n} y u_{n}^{*}\right\|_{\infty}^{1-1 / p}\left\|x-u_{n} y u_{n}^{*}\right\|_{1}^{1 / p} \rightarrow 0, \quad 1 \leq p \leq \infty,
$$

the lemma is proved. 
REMARK 4.2. Let $\mathscr{M}$ be a semifinite factor and $x, y \in \tilde{\mathfrak{S}}_{s a}$. Then $x \approx y$ if and only if $x$ is in the closure of $\mathscr{U}(y)$ in the measure topology. In fact, if the latter holds, then it follows from [43] that $x_{+}$(resp. $x_{-}$) is in the closure of $\mathscr{U}\left(y_{+}\right)$(resp. $\mathscr{U}\left(y_{-}\right)$) in the measure topology. Hence $x \approx y$ by [22, Theorem 3.4(1)]. The converse is obvious from Lemma 4.1. Moreover, by [29, Theorem 4.4] (also [43]) and [22, Theorem 3.4(2)], if $y \in L^{p}(\mathscr{M})$ where $1 \leq p \leq \infty$, then $x \approx y$ if and only if $x$ is in the $\|\cdot\|_{p}$-closure of $\mathscr{U}(y)$.

We are now in a position to obtain the theorems.

THEOREM 4.3. Assume that $\mathscr{M}$ is a finite factor. If $x, y \in \tilde{\mathscr{M}}_{s a}$, then for $1 \leq p \leq \infty$

$$
\begin{aligned}
& \inf _{u \in \mathscr{U}}\left\|x-u y u^{*}\right\|_{p}=\|\lambda(x)-\lambda(y)\|_{p}, \\
& \sup _{u \in \mathscr{U}}\left\|x-u y u^{*}\right\|_{p}=\|\lambda(x)-\check{\lambda}(y)\|_{p} .
\end{aligned}
$$

Furthermore there exist $y^{\prime}, y^{\prime \prime} \in \tilde{\mathscr{M}}_{s a}$ such that $y^{\prime} \approx y, y^{\prime \prime} \approx y$, $\left\|x-y^{\prime}\right\|_{p}=\|\lambda(x)-\lambda(y)\|_{p}$ and $\left\|x-y^{\prime \prime}\right\|_{p}=\|\lambda(x)-\check{\lambda}(y)\|_{p}$ for all $1 \leq p \leq \infty$.

Proof. For $1 \leq p<\infty$, because $\lambda \mapsto \lambda^{p}$ is an increasing convex function on $[0, \infty)$, by Theorem 3.1(1) and [38, Theorem 3.1] we have

$$
|\lambda(x)-\lambda(y)|^{p} \prec|\lambda(x-y)|^{p} \prec|\lambda(x)-\check{\lambda}(y)|^{p},
$$

so that

$$
\|\lambda(x)-\lambda(y)\|_{p} \leq\|x-y\|_{p} \leq\|\lambda(x)-\check{\lambda}(y)\|_{p} .
$$

The above inequalities are valid also for $p=\infty$ by Theorem 3.1(1). Since $\lambda(y)=\lambda\left(u y u^{*}\right)$ and $\check{\lambda}(y)=\check{\lambda}\left(u y u^{*}\right)$ for $u \in \mathscr{U}$,

$$
\begin{aligned}
& \inf _{u \in \mathscr{U}}\left\|x-u y u^{*}\right\|_{p} \geq\|\lambda(x)-\lambda(y)\|_{p}, \\
& \sup _{u \in \mathscr{U}}\left\|x-u y u^{*}\right\|_{p} \leq\|\lambda(x)-\check{\lambda}(y)\|_{p} .
\end{aligned}
$$

In the following, assume that $\mathscr{M}$ is a factor of type $\mathrm{II}_{1}$ and $\tau(1)=1$ (the theorem is well known when $\mathscr{M}=\mathbf{M}_{n}$ ). Let $x=\int_{-\infty}^{\infty} s d e_{s}$ be the spectral decomposition of $x$ (i.e. $e_{s}=e_{(-\infty, s]}(x)$ ), and define $\tilde{x}=$ $\int_{-\infty}^{\infty} \tau\left(e_{(s, \infty)}(x)\right) d e_{s}$. Then $\tilde{x}$ is an operator in $\mathscr{M}$ with $0 \leq \tilde{x} \leq 1$. Let $\tilde{x}=\int_{0}^{1} t d \tilde{e}_{t}$ be the spectral decomposition of $\tilde{x}$. Since

$$
\tilde{e}_{t}=e_{\left[\lambda_{t}(x), \infty\right)}(x), \quad 0<t<1,
$$


we get $x=\int_{0}^{1} \lambda_{t}(x) d \tilde{e}_{t}$ (the Schmidt decomposition [35]). Now let $r_{1}, r_{2}, \ldots$ be an enumeration of all $r \in \mathbf{R}$ with $e_{\{r\}}(x) \neq 0$. Let $t_{k}=$ $\tau\left(e_{\left(r_{k}, \infty\right)}(x)\right)$ and $t_{k}^{\prime}=\tau\left(e_{\left[r_{k}, \infty\right)}(x)\right)$. Then

$$
\tau\left(\tilde{e}_{t}\right)= \begin{cases}t, & t \in[0,1] \backslash \bigcup_{k}\left[t_{k}, t_{k}^{\prime}\right), \\ t_{k}^{\prime}, & t \in\left[t_{k}, t_{k}^{\prime}\right) .\end{cases}
$$

For each $k$, we can choose an increasing family $\left\{p_{t}^{(k)}: 0 \leq t \leq t_{k}^{\prime}-t_{k}\right\}$ of projections such that $p_{t_{k}^{\prime}-t_{k}}^{(k)}=e_{\left\{r_{k}\right\}}(x)$ and $\tau\left(p_{t}^{(k)}\right)=t$ for $0 \leq t \leq$ $t_{k}^{\prime}-t_{k}$. Letting

$$
\bar{e}_{t}= \begin{cases}\tilde{e}_{t}, & t \in[0,1] \backslash \bigcup_{k}\left[t_{k}, t_{k}^{\prime}\right), \\ e_{\left(r_{k}, \infty\right)}(x)+p_{t-t_{k}}^{(k)}, & t \in\left[t_{k}, t_{k}^{\prime}\right),\end{cases}
$$

we have an increasing family $\left\{\bar{e}_{t}: 0 \leq t \leq 1\right\}$ such that $\tau\left(\bar{e}_{t}\right)=t$ for $0 \leq t \leq 1$ and $x=\int_{0}^{1} \lambda_{t}(x) d \bar{e}_{t}$. Define $y^{\prime}=\int_{0}^{1} \lambda_{t}(y) d \bar{e}_{t}$ and $y^{\prime \prime}=\int_{0}^{1} \check{\lambda}_{t}(y) d \bar{e}_{t}$. Since $\lambda\left(y^{\prime}\right)=\lambda(y)$ and $\lambda\left(y^{\prime \prime}\right)=(\check{\lambda}(y))^{*}=\lambda(y)$, by Lemma 4.1 there are sequences $\left\{u_{n}\right\}$ and $\left\{v_{n}\right\}$ in $\mathscr{U}$ such that $\left\|y^{\prime}-u_{n} y u_{n}^{*}\right\|_{p} \rightarrow 0$ and $\left\|y^{\prime \prime}-v_{n} y v_{n}^{*}\right\|_{p} \rightarrow 0$ for $1 \leq p \leq \infty$. Hence

$$
\begin{aligned}
& \inf _{u \in \mathscr{U}}\left\|x-u y u^{*}\right\|_{p} \leq \liminf _{n \rightarrow \infty}\left\|x-u_{n} y u_{n}^{*}\right\|_{p} \leq\left\|x-y^{\prime}\right\|_{p}, \\
& \sup _{u \in \mathscr{U}}\left\|x-u y u^{*}\right\|_{p} \geq \limsup _{n \rightarrow \infty}\left\|x-v_{n} y v_{n}^{*}\right\|_{p} \geq\left\|x-y^{\prime \prime}\right\|_{p} .
\end{aligned}
$$

Moreover it is immediate from definitions that

$$
\left\|x-y^{\prime}\right\|_{p}=\|\lambda(x)-\lambda(y)\|_{p} \text { and }\left\|x-y^{\prime \prime}\right\|_{p}=\|\lambda(x)-\check{\lambda}(y)\|_{p} .
$$

These complete the proof.

THEOREM 4.4. Assume that $\mathscr{M}$ is an infinite semifinite factor. If $x, y \in \tilde{\mathfrak{S}}_{s a}$, then for $1 \leq p \leq \infty$

$$
\begin{aligned}
& \inf _{u \in \mathscr{U}}\left\|x-u y u^{*}\right\|_{p}=\|\lambda(x)-\lambda(y)\|_{p}, \\
& \sup _{u \in \mathscr{U}}\left\|x-u y u^{*}\right\|_{p}=\|\lambda(x)-\check{\lambda}(y)\|_{p} .
\end{aligned}
$$

Furthermore there exist $x^{\prime}, y^{\prime}, y^{\prime \prime} \in \tilde{\mathfrak{S}}_{s a}$ such that $x^{\prime} \approx x, y^{\prime} \approx y, y^{\prime \prime} \approx$ $y,\left\|x^{\prime}-y^{\prime}\right\|_{p}=\|\lambda(x)-\lambda(y)\|_{p}$ and $\left\|x^{\prime}-y^{\prime \prime}\right\|_{p}=\|\lambda(x)-\grave{\lambda}(y)\|_{p}$ for all $1 \leq p \leq \infty$.

Proof. By Theorem 3.1(2) and [38, Theorem 3.1], we have for $1 \leq$ $p \leq \infty$

$$
\|\lambda(x)-\lambda(y)\|_{p} \leq\|x-y\|_{p} \leq\|\lambda(x)-\check{\lambda}(y)\|_{p}
$$


and thus

$$
\begin{aligned}
& \inf _{u \in \mathscr{U}}\left\|x-u y u^{*}\right\|_{p} \geq\|\lambda(x)-\lambda(y)\|_{p}, \\
& \sup _{u \in \mathscr{U}}\left\|x-u y u^{*}\right\|_{p} \leq\|\lambda(x)-\check{\lambda}(y)\|_{p} .
\end{aligned}
$$

Now assume that $\mathscr{M}$ is a factor of type $\mathrm{II}_{\infty}$ (the type $\mathrm{I}_{\infty}$ case is analogously proved). We choose two increasing families $\left\{e_{t}: t \geq 0\right\}$ and $\left\{f_{t}: t \geq 0\right\}$ of projections such that $\tau\left(e_{t}\right)=\tau\left(f_{t}\right)=t$ for $t \geq 0$ and $\bigvee_{t \geq 0} e_{t} \perp \bigvee_{t \geq 0} f_{t}$. Define

$$
\begin{aligned}
x^{\prime} & =\int_{0}^{\infty} \mu_{t}\left(x_{+}\right) d e_{t}-\int_{0}^{\infty} \mu_{t}\left(x_{-}\right) d f_{t}, \\
y^{\prime} & =\int_{0}^{\infty} \mu_{t}\left(y_{+}\right) d e_{t}-\int_{0}^{\infty} \mu_{t}\left(y_{-}\right) d f_{t}, \\
y^{\prime \prime} & =\int_{0}^{\infty} \mu_{t}\left(y_{+}\right) d f_{t}-\int_{0}^{\infty} \mu_{t}\left(y_{-}\right) d e_{t} .
\end{aligned}
$$

Then $\left\|x^{\prime}-y^{\prime}\right\|_{p}=\|\lambda(x)-\lambda(y)\|_{p}$ and $\left\|x^{\prime}-y^{\prime \prime}\right\|_{p}=\|\lambda(x)-\check{\lambda}(y)\|_{p}$, $1 \leq p \leq \infty$. Since $\lambda\left(x^{\prime}\right)=\boldsymbol{\lambda}(x)$ and $\boldsymbol{\lambda}\left(y^{\prime}\right)=\boldsymbol{\lambda}\left(y^{\prime \prime}\right)=\boldsymbol{\lambda}(y)$, by Lemma 4.1 there are sequences $\left\{u_{n}\right\},\left\{v_{n}\right\}$ and $\left\{w_{n}\right\}$ in $\mathscr{U}$ such that $\left\|x^{\prime}-u_{n} x u_{n}^{*}\right\|_{p} \rightarrow 0,\left\|y^{\prime}-v_{n} y v_{n}^{*}\right\|_{p} \rightarrow 0$ and $\left\|y^{\prime \prime}-w_{n} y w_{n}^{*}\right\|_{p} \rightarrow 0$ for $1 \leq p \leq \infty$. Therefore

$$
\begin{aligned}
& \inf _{u \in \mathscr{U}}\left\|x-u y u^{*}\right\|_{p} \leq \liminf _{n \rightarrow \infty}\left\|u_{n} x u_{n}^{*}-v_{n} y v_{n}^{*}\right\|_{p} \leq\left\|x^{\prime}-y^{\prime}\right\|_{p}, \\
& \sup _{u \in \mathscr{C}}\left\|x-u y u^{*}\right\|_{p} \geq \limsup _{n \rightarrow \infty}\left\|u_{n} x u_{n}^{*}-w_{n} y w_{n}^{*}\right\|_{p} \geq\left\|x^{\prime}-y^{\prime \prime}\right\|_{p},
\end{aligned}
$$

completing the proof.

REMARK 4.5. (1) In particular when $x, y \in \tilde{\mathfrak{S}}_{+}$, the formulas in Theorem 4.4 are written as follows: for $1 \leq p \leq \infty$

$$
\begin{aligned}
& \inf _{u \in \mathscr{U}}\left\|x-u y u^{*}\right\|_{p}=\|\mu(x)-\mu(y)\|_{p}, \\
& \sup _{u \in \mathscr{U}}\left\|x-u y u^{*}\right\|_{p}=\left(\|x\|_{p}^{p}+\|y\|_{p}^{p}\right)^{1 / p} .
\end{aligned}
$$

Here and in Theorem 4.6 below, $\left(\|x\|_{p}^{p}+\|y\|_{p}^{p}\right)^{1 / p}$ when $p=\infty$ means $\max \left\{\|x\|_{\infty},\|y\|_{\infty}\right\}$.

(2) Under the assumption of Theorem 4.4, it is not difficult to see that for each $x, y \in \tilde{\mathfrak{S}}_{s a}$ there exists either $x^{\prime}$ such that $x^{\prime} \approx x$ and $\left\|x^{\prime}-y\right\|_{1}=\|\lambda(x)-\lambda(y)\|_{1}$ (resp. $\left.\left\|x^{\prime}-y\right\|_{\infty}=\|\lambda(x)-\check{\lambda}(y)\|_{\infty}\right)$ or $y^{\prime}$ such that $y^{\prime} \approx y$ and $\left\|x-y^{\prime}\right\|_{1}=\|\lambda(x)-\lambda(y)\|_{1}$ (resp. $\left\|x-y^{\prime}\right\|_{\infty}=$ $\left.\|\lambda(x)-\check{\lambda}(y)\|_{\infty}\right)$. However, for $1<p \leq \infty$ (resp. $\left.1 \leq p<\infty\right)$, there are $x, y \in \tilde{\mathfrak{S}}_{s a}$ for which we obtain neither $x^{\prime}$ such that $x^{\prime} \approx x$ 
and $\left\|x^{\prime}-y\right\|_{p}=\|\lambda(x)-\lambda(y)\|_{p}$ (resp. $\left.\left\|x^{\prime}-y\right\|_{p}=\|\lambda(x)-\check{\lambda}(y)\|_{p}\right)$ nor $y^{\prime}$ such that $y^{\prime} \approx y$ and $\left\|x-y^{\prime}\right\|_{p}=\|\boldsymbol{\lambda}(x)-\lambda(y)\|_{p}$ (resp. $\left.\left\|x-y^{\prime}\right\|_{p}=\|\lambda(x)-\check{\lambda}(y)\|_{p}\right)$. For instance, for $1<p<\infty$, let $x$ be strictly positive and $y$ be strictly negative in $L^{p}(\mathscr{M})$. Suppose that $x^{\prime} \approx x$ and $\left\|x^{\prime}-y\right\|_{p}=\|\lambda(x)-\lambda(y)\|_{p}$. Then $\left\|x^{\prime}-y\right\|_{p}^{p}=\left\|x^{\prime}\right\|_{p}^{p}+\|y\|_{p}^{p}$, showing $x^{\prime} y=0$ by [29, Proposition 6.3], so we get $x=0$, a contradiction. The argument for $y^{\prime}$ is analogous.

(3) We note that $\delta(x, y)=\|\lambda(x)-\lambda(y)\|_{\infty}$ for $x, y \in \tilde{\mathscr{M}}_{s a}$ with $\tau(1)<\infty$ and $\delta(x, y)=\|\lambda(x)-\lambda(y)\|_{\infty}$ for $x, y \in \tilde{\mathfrak{S}}_{s a}$ with $\tau(1)=\infty$ (the definition of $\delta(x, y)$ is available for $\left.x, y \in \tilde{\mathscr{M}}_{s a}\right)$. These equalities follow from Theorem 2.1(3), 4.3 and 4.4 when $x$ and $y$ are bounded and $\mathscr{M}$ is a factor. But it is rather easy to check them directly without the factor assumption.

Ando and Bhatia [3] obtained some inequalities on $L^{p}$-distances for Hermitian and skew-Hermitian matrices by a method of majorization based on the Lidskii-Wielandt theorem. In the following theorem, by the same method as [3], we estimate $L^{p}$-distances between unitary orbits of $\tau$-measurable selfadjoint and skew-adjoint operators. Here the bounds for $\inf _{u \in \mathscr{U}}\left\|x-i u y u^{*}\right\|_{p}$ in (1) and $\sup _{u \in \mathscr{U}}\left\|x-i u y u^{*}\right\|_{p}$ in (2) are best even for $2 \times 2$ matrices as noted in [3].

THEOREM 4.6. Assume that $\mathscr{M}$ is a semifinite factor and $x, y \in \tilde{\mathfrak{S}}_{s a}$. (1) For $0<p \leq 2$,

$$
\begin{gathered}
\inf _{u \in \mathscr{U}}\left\|x-i u y u^{*}\right\|_{p} \geq 2^{1 / 2-1 / p}\left\|\left\{\mu(x)^{2}+\mu(y)^{2}\right\}^{1 / 2}\right\|_{p}, \\
\sup _{u \in \mathscr{U}}\left\|x-i u y u^{*}\right\|_{p}= \begin{cases}\left\|\left\{\lambda(|x|)^{2}+\check{\lambda}(|y|)^{2}\right\}^{1 / 2}\right\|_{p} & \text { if } \mathscr{M} \text { is finite, } \\
\left(\|x\|_{p}^{p}+\|y\|_{p}^{p}\right)^{1 / p} & \text { if } \mathscr{M} \text { is infinite. }\end{cases}
\end{gathered}
$$

(2) For $2 \leq p \leq \infty$,

$$
\begin{gathered}
\inf _{u \in \mathscr{U}}\left\|x-i u y u^{*}\right\|_{p}= \begin{cases}\left\|\left\{\lambda(|x|)^{2}+\check{\lambda}(|y|)^{2}\right\}^{1 / 2}\right\|_{p} & \text { if } \mathscr{M} \text { is finite, } \\
\left(\|x\|_{p}^{p}+\|y\|_{p}^{p}\right)^{1 / p} & \text { if } \mathscr{M} \text { is infinite, }\end{cases} \\
\sup _{u \in \mathscr{U}}\left\|x-i u y u^{*}\right\|_{p} \leq 2^{1 / 2-1 / p}\left\|\left\{\mu(x)^{2}+\mu(y)^{2}\right\}^{1 / 2}\right\|_{p} .
\end{gathered}
$$

Proof. If either $x$ or $y$ is not in $L^{p}(\mathscr{M})$, then the desired equalities and inequalities hold with the both sides being $\infty$. So we assume $x, y \in L^{p}(\mathscr{M})_{s a}$. First let $\mathscr{M}$ be finite and $z=x-i y$. By the extension [24] of the Lidskii-Wielandt theorem, we get

$$
\begin{gathered}
\lambda(|x|)^{2}+\check{\lambda}(|y|)^{2} \prec \lambda\left(x^{2}+y^{2}\right) \prec \lambda(|x|)^{2}+\lambda(|y|)^{2}, \\
\left\{\lambda(|z|)^{2}+\check{\lambda}(|z|)^{2}\right\} / 2 \prec \lambda\left(x^{2}+y^{2}\right) \prec \lambda(|z|)^{2},
\end{gathered}
$$


since $x^{2}+y^{2}=\left(z^{*} z+z z^{*}\right) / 2$ and $\lambda\left(z^{*} z\right)=\lambda\left(z z^{*}\right)=\lambda(|z|)^{2}($ see $[24])$. Hence

$$
\begin{gathered}
\lambda(|x|)^{2}+\check{\lambda}(|y|)^{2} \prec \lambda(|z|)^{2}, \\
\lambda(|z|)^{2}+\check{\lambda}(|z|)^{2} \prec 2\left\{\lambda(|x|)^{2}+\lambda(|y|)^{2}\right\} .
\end{gathered}
$$

Because $\lambda \mapsto \lambda^{p / 2}$ on $[0, \infty)$ is concave when $0<p \leq 2$ and convex when $2 \leq p<\infty$, by [11, Theorem 2.5] we have for $0<p \leq 2$

$$
\begin{gathered}
\left\|\left\{\lambda(|x|)^{2}+\check{\lambda}(|y|)^{2}\right\}^{1 / 2}\right\|_{p} \geq\|\lambda(|z|)\|_{p}, \\
\left\|\left\{\lambda(|z|)^{2}+\check{\lambda}(|z|)^{2}\right\}^{1 / 2}\right\|_{p} \geq 2^{1 / 2}\left\|\left\{\lambda(|x|)^{2}+\lambda(|y|)^{2}\right\}^{1 / 2}\right\|_{p},
\end{gathered}
$$

and for $2 \leq p<\infty$ the reversed inequalities which are valid also for $p=\infty$. Moreover we have for $0<p \leq 2$

$$
\left\|\left\{\lambda(|z|)^{2}+\check{\lambda}(|z|)^{2}\right\}^{1 / 2}\right\|_{p} \leq\left\{\|\lambda(|z|)\|_{p}^{p}+\|\check{\lambda}(|z|)\|_{p}^{p}\right\}^{1 / p},
$$

and for $2 \leq p \leq \infty$ the reversed inequality. Therefore, since $\|\lambda(|z|)\|_{p}$ $=\|\check{\lambda}(|z|)\|_{p}=\|z\|_{p}$, the following inequalities are obtained: for $0<$ $p \leq 2$

$$
2^{1 / 2-1 / p}\left\|\left\{\lambda(|x|)^{2}+\lambda(|y|)^{2}\right\}^{1 / 2}\right\|_{p} \leq\|z\|_{p} \leq\left\|\left\{\lambda(|x|)^{2}+\check{\lambda}(|y|)^{2}\right\}^{1 / 2}\right\|_{p},
$$

and for $2 \leq p \leq \infty$

$$
\left\|\left\{\lambda(|x|)^{2}+\check{\lambda}(|y|)^{2}\right\}^{1 / 2}\right\|_{p} \leq\|z\|_{p} \leq 2^{1 / 2-1 / p}\left\|\left\{\lambda(|x|)^{2}+\lambda(|y|)^{2}\right\}^{1 / 2}\right\|_{p} .
$$

On the other hand, it is readily seen that if $x$ and $y$ have finite spectra, then there exist $x^{\prime}, y^{\prime} \in \mathscr{M}_{s a}$ such that $x^{\prime} \approx x, y^{\prime} \approx y$ and

$$
\left\|x^{\prime}-i y^{\prime}\right\|_{p}=\left\|\left\{\lambda(|x|)^{2}+\check{\lambda}(|y|)^{2}\right\}^{1 / 2}\right\|_{p} \text {. }
$$

Hence, approximating $x$ and $y$ by operators with finite spectra, we get

$$
\inf _{u \in \mathscr{U}}\left\|x-i u y u^{*}\right\|_{p} \leq\left\|\left\{\lambda(|x|)^{2}+\check{\lambda}(|y|)^{2}\right\}^{1 / 2}\right\|_{p} \leq \sup _{u \in \mathscr{C}}\left\|x-i u y u^{*}\right\|_{p},
$$

so that the theorem in the finite case is proved.

Next let $\mathscr{M}$ be infinite. For $n \geq 1$, let $x_{n}, y_{n}$ and $e$ be as in the proof of Theorem 3.1(2). Since $x, y \in L^{p}(\mathscr{M})$, we get $\left\|x_{n}-x\right\|_{p} \rightarrow 0$, $\left\|y_{n}-y\right\|_{p} \rightarrow 0$, and hence by [24, Corollary 3]

$$
\begin{gathered}
\lim _{n \rightarrow \infty}\left\|\left\{\lambda\left(\left|x_{n}\right|\right)^{2}+\lambda\left(\left|y_{n}\right|\right)^{2}\right\}^{1 / 2}\right\|_{p} \\
=\lim _{n \rightarrow \infty}\left\|\left\{\mu\left(x_{n}\right)^{2}+\mu\left(y_{n}\right)^{2}\right\}^{1 / 2}\right\|_{p}=\left\|\left\{\mu(x)^{2}+\mu(y)^{2}\right\}^{1 / 2}\right\|_{p}, \\
\lim _{n \rightarrow \infty}\left\|\left\{\lambda\left(\left|x_{n}\right|\right)^{2}+\check{\lambda}\left(\left|y_{n}\right|\right)^{2}\right\}^{1 / 2}\right\|_{p} \\
=\lim _{n \rightarrow \infty}\left(\left\|x_{n}\right\|_{p}^{p}+\left\|y_{n}\right\|_{p}^{p}\right)=\left(\|x\|_{p}^{p}+\|y\|_{p}^{p}\right)^{1 / p},
\end{gathered}
$$


where $\lambda\left(\left|x_{n}\right|\right)$ and others are defined on $(0, \tau(e))$. Thus the desired estimates are obtained by applying the assertions in the finite case to $x_{n}, y_{n} \in L^{p}\left(\mathscr{M}_{e}\right)_{s a}$ and then by passing to the limits as $n \rightarrow \infty$.

5. $L^{p}$-distance between unitary orbits in Haagerup $L^{p}$-spaces. In this section, we shall obtain some formulas of $L^{p}$-distance and anti- $L^{p}$ distance between unitary orbits in Haagerup $L^{p}$-spaces, i.e. noncommutative $L^{p}$-spaces over general von Neumann algebras introduced in [19].

We begin with a very brief survey on Haagerup $L^{p}$-spaces (see [42] for details). Let $\mathscr{M}$ be a general von Neumann algebra with a faithful normal semifinite weight $\varphi_{0}$. Denote by $\mathscr{N}$ the crossed product $\mathscr{M} \rtimes_{\sigma^{\varphi_{0}}} \mathbf{R}$ which admits the canonical faithful normal semifinite trace $\tau$ and the dual action $\theta_{s}, s \in \mathbf{R}$, satisfying $\tau \circ \theta_{s}=e^{-s} \tau, s \in \mathbf{R}$. For $0<p \leq \infty$, the Haagerup $L^{p}$-space $L^{p}(\mathscr{M})=L^{p}\left(\mathscr{M} ; \varphi_{0}\right)$ is defined by

$$
L^{p}(\mathscr{M})=\left\{x \in \tilde{\mathscr{N}}: \theta_{s}(x)=e^{-s / p} x, s \in \mathbf{R}\right\} .
$$

Here $\mathscr{M}=L^{\infty}(\mathscr{M})$. For each $\psi \in \mathscr{M}_{*}^{+}$, a unique $h_{\psi} \in \tilde{\mathscr{N}}_{+}$is given by $\tilde{\psi}=\tau\left(h_{\psi} \cdot\right)$ where $\tilde{\psi}$ is the dual weight of $\psi$. The mapping $\psi \mapsto h_{\psi}$ is extended to a linear bijection from $\mathscr{M}_{*}$ onto $L^{1}(\mathscr{M})$, and so the linear functional $\operatorname{tr}$ on $L^{1}(\mathscr{M})$ is defined by $\operatorname{tr}\left(h_{\psi}\right)=\psi(1), \psi \in \mathscr{M}_{*}$. For $0<p<\infty$, the Haagerup (quasi-)norm $\|x\|_{p}$ of $x \in L^{p}(\mathscr{M})$ is defined by $\|x\|_{p}=\operatorname{tr}\left(|x|^{p}\right)^{1 / p}$. When $1 \leq p<\infty, L^{p}(\mathscr{M})$ is a Banach space with the norm $\|\cdot\|_{p}$, and its dual Banach space is $L^{q}(\mathscr{M})$ where $1 / p+1 / q=1$ by the following duality:

$$
\langle x, y\rangle=\operatorname{tr}(x y)(=\operatorname{tr}(y x)), \quad x \in L^{p}(\mathscr{M}), y \in L^{q}(\mathscr{M}) .
$$

In particular, $\mathscr{M}_{*} \cong L^{1}(\mathscr{M})$ by the isometry $\psi \mapsto h_{\psi}$.

Let $L^{p}(\mathscr{M})_{s a}$ (resp. $\left.L^{p}(\mathscr{M})_{+}\right)$denote $L^{p}(\mathscr{M}) \cap \tilde{\mathscr{N}}_{s} a\left(\right.$ resp. $L^{p}(\mathscr{M}) \cap$ $\left.\tilde{\mathscr{N}}_{+}\right)$. Note that the support projection $s(x)$ of each $x \in L^{p}(\mathscr{M})_{+}$is in $\mathscr{M}$. The unitary orbit $\mathscr{U}(x)$ of $x \in L^{p}(\mathscr{M})$ is given by $\mathscr{U}(x)=$ $\left\{u x u^{*}: u \in \mathscr{U}\right\}$ where $\mathscr{U}$ is the unitaries in $\mathscr{M}$. Then $\mathscr{U}(x)$ is included in $L^{p}(\mathscr{M})$ if $x \in L^{p}(\mathscr{M})$. The space $L^{p}(\mathscr{M})$, together with $L^{p}(\mathscr{M})_{+}$, is independent of the choice of $\varphi_{0}$ up to isomorphism. Furthermore, when $\mathscr{M}$ is semifinite with a faithful normal semifinite trace $\tau$, the Haagerup $L^{p}$-space $L^{p}\left(\mathscr{M} ; \varphi_{0}=\tau\right)$ coincides with $L^{p}(\mathscr{M} ; \tau)$ in the previous sense.

The next lemma gives general bounds for the distance $\|x-y\|_{p}$ between $x, y \in L^{p}(\mathscr{M})_{s a}$. The first inequality extends the inequality established in [29, Lemma 3.3] (also [17, Lemma 5.1]). 
LeMMA 5.1. If $1 \leq p<\infty$ and $x, y \in L^{p}(\mathscr{M})_{s a}$, then

$$
\begin{aligned}
& \left\{\left|\left\|x_{+}\right\|_{p}-\left\|y_{+}\right\|_{p}\right|^{p}+\left|\left\|x_{-}\right\|_{p}-\left\|y_{-}\right\|_{p}\right|^{p}\right\}^{1 / p} \\
& \quad \leq\|x-y\|_{p} \leq\left\{\left(\left\|x_{+}\right\|_{p}+\left\|y_{-}\right\|_{p}\right)^{p}+\left(\left\|x_{-}\right\|_{p}+\left\|y_{+}\right\|_{p}\right)^{p}\right\}^{1 / p} .
\end{aligned}
$$

Proof. First let $1<p<\infty$. Since $L^{p}(\mathscr{M})_{s a} \subseteq \tilde{\mathfrak{S}}_{s a}$ where $\tilde{\mathfrak{S}}$ is with respect to $(\mathscr{N}, \tau)$, Theorem $3.1(2)$ shows that

$$
\begin{aligned}
\int_{0}^{1}|\lambda(x)-\lambda(y)|^{*}(t) d t & \leq \int_{0}^{1}|\lambda(x-y)|^{*}(t) d t \\
& \leq \int_{0}^{1}|\boldsymbol{\lambda}(x)-\check{\lambda}(y)|^{*}(t) d t .
\end{aligned}
$$

By [17, Lemma 4.8],

$$
\begin{array}{r}
\left|\boldsymbol{\lambda}_{t}(x)-\lambda_{t}(y)\right|= \begin{cases}t^{-1 / p}\left|\left\|x_{+}\right\|_{p}-\left\|y_{+}\right\|_{p}\right|, & t>0, \\
(-t)^{-1 / p}\left|\left\|x_{-}\right\|_{p}-\left\|y_{-}\right\|_{p}\right|, & t<0,\end{cases} \\
\left|\boldsymbol{\lambda}_{t}(x)-\check{\boldsymbol{\lambda}}_{t}(y)\right|= \begin{cases}t^{-1 / p}\left(\left\|x_{+}\right\|_{p}+\left\|y_{-}\right\|_{p}\right), & t>0, \\
(-t)^{-1 / p}\left(\left\|x_{-}\right\|_{p}+\left\|y_{+}\right\|_{p}\right), & t<0 .\end{cases}
\end{array}
$$

Hence, from an easy computation, we get

$$
\begin{aligned}
& \int_{0}^{1}|\lambda(x)-\lambda(y)|^{*}(t) d t \\
& =\left\{| | \left|x_{+}\left\|_{p}-\right\| y_{+}\left\|\left._{p}\right|^{p}+||\left|x_{-}\left\|_{p}-\right\| y_{-} \|_{p}\right|^{p}\right\}^{1 / p},\right.\right. \\
& \int_{0}^{1}|\boldsymbol{\lambda}(x)-\check{\lambda}(y)|^{*}(t) d t \\
& =\left\{\left(\left\|x_{+}\right\|_{p}+\left\|y_{-}\right\|_{p}\right)^{p}+\left(\left\|x_{-}\right\|_{p}+\left\|y_{+}\right\|_{p}\right)^{p}\right\}^{1 / p} .
\end{aligned}
$$

Moreover $\int_{0}^{1}|\lambda(x-y)|^{*}(t) d t=\|x-y\|_{p}$ by Lemma 3.2(2) and [17, Lemma 4.8]. These imply the desired inequalities for $1<p<\infty$.

When $p=1$, the second inequality is obvious. If $\left\|x_{+}\right\|_{1} \geq\left\|y_{+}\right\|_{1}$ and $\left\|x_{-}\right\|_{1} \geq\left\|y_{-}\right\|_{1}$, then

$$
\left|\left\|x_{+}\right\|_{1}-\left\|y_{+}\right\|_{1}\right|+\left|\left\|x_{-}\right\|_{1}-\left\|y_{-}\right\|_{1}\right|=\|x\|_{1}-\|y\|_{1} \leq\|x-y\|_{1} .
$$

If $\left\|x_{+}\right\|_{1} \geq\left\|y_{+}\right\|_{1}$ and $\left\|x_{-}\right\|_{1} \leq\left\|y_{-}\right\|_{1}$, then

$$
\begin{aligned}
& \left|\left\|x_{+}\right\|_{1}-\left\|y_{+}\right\|_{1}\right|+\left|\left\|x_{-}\right\|_{1}-\left\|y_{-}\right\|_{1}\right| \\
& \quad=\left\|x_{+}\right\|_{1}-\left\|x_{-}\right\|_{1}-\left\|y_{+}\right\|_{1}+\left\|y_{-}\right\|_{1}=\operatorname{tr}(x-y) \leq\|x-y\|_{1} .
\end{aligned}
$$

Hence the first inequality for $p=1$ is proved.

The next lemma is useful to estimate $L^{p}$-distances between $\mathscr{U}(x)$ and $\mathscr{U}(y)$ for $x, y \in L^{p}(\mathscr{M})_{s a}$ when $\mathscr{M}$ is a type $\mathrm{III}_{1}$ factor. 
Lemma 5.2. Assume that $\mathscr{M}$ is a factor of type $\mathrm{III}_{1}$. Let $e$ be a projection in $\mathscr{M}$ and $x, y \in L^{p}(\mathscr{M})_{+}, 0<p<\infty$, with $s(x) \leq e$ and $s(y) \leq e$. If $\|x\|_{p}=\|y\|_{p}$, then

$$
\inf _{u \in \mathscr{U}_{e}}\left\|x-u y u^{*}\right\|_{p}=0,
$$

where $\mathscr{U}_{e}$ is the unitaries in $\mathscr{M}_{e}$.

Proof. First assume $1 \leq p<\infty$. By the generalized Powers-Størmer inequality in the appendix, it follows that

$$
\left\|x-u y u^{*}\right\|_{p} \leq\left\|x^{p}-\left(u y u^{*}\right)^{p}\right\|_{1}^{1 / p}=\left\|x^{p}-u y^{p} u^{*}\right\|_{1}^{1 / p}
$$

for all $u \in \mathscr{U}_{e}$. Because $\mathscr{M}_{e}$ is a factor of type $\mathrm{III}_{1}$ and $\left\|x^{p}\right\|_{1}=\left\|y^{p}\right\|_{1}$, considering $x^{p}, y^{p} \in L^{1}(\mathscr{M})_{+}$as elements in $\left(\mathscr{M}_{e}\right)_{*}^{+}$we get

$$
\inf _{u \in \mathscr{U}_{e}}\left\|x^{p}-u y^{p} u^{*}\right\|_{1}=0
$$

by [13, Theorem 4] which remains valid for any factor of type $\mathrm{III}_{1}$. Hence the desired conclusion is verified when $1 \leq p<\infty$. Next assume $0<p<1$. By [17, Theorem 4.9(iii)] and Hölder's inequality (see [17, Theorem 4.9(i)]), we get

$$
\begin{aligned}
& 2^{p}\|x-y\|_{p}^{p} \\
& \quad=\left\|\left(x^{1 / 2}+y^{1 / 2}\right)\left(x^{1 / 2}-y^{1 / 2}\right)+\left(x^{1 / 2}-y^{1 / 2}\right)\left(x^{1 / 2}+y^{1 / 2}\right)\right\|_{p}^{p} \\
& \quad \leq\left\|\left(x^{1 / 2}+y^{1 / 2}\right)\left(x^{1 / 2}-y^{1 / 2}\right)\right\|_{p}^{p}+\left\|\left(x^{1 / 2}-y^{1 / 2}\right)\left(x^{1 / 2}+y^{1 / 2}\right)\right\|_{p}^{p} \\
& \quad \leq 2\left\|x^{1 / 2}+y^{1 / 2}\right\|_{2 p}^{p}\left\|x^{1 / 2}-y^{1 / 2}\right\|_{2 p}^{p} \\
& \quad \leq 4\|x\|_{p}^{p / 2}\left\|x^{1 / 2}-y^{1 / 2}\right\|_{2 p}^{p} .
\end{aligned}
$$

This implies that the validity of the conclusion for $p$ follows from that for $2 p$. Thus the lemma is proved.

THeOREM 5.3. Assume that $\mathscr{M}$ is a factor of type $\mathrm{III}_{1}$. If $1 \leq p<\infty$ and $x, y \in L^{p}(\mathscr{M})_{s a}$, then

$$
\begin{aligned}
& \inf _{u \in \mathscr{U}}\left\|x-u y u^{*}\right\|_{p}=\left\{\left|\left\|x_{+}\right\|_{p}-\left\|y_{+}\right\|_{p}\right|^{p}+\left|\left\|x_{-}\right\|_{p}-\left\|y_{-}\right\|_{p}\right|^{p}\right\}^{1 / p}, \\
& \sup _{u \in \mathscr{U}}\left\|x-u y u^{*}\right\|_{p}=\left\{\left(\left\|x_{+}\right\|_{p}+\left\|y_{-}\right\|_{p}\right)^{p}+\left(\left\|x_{-}\right\|_{p}+\left\|y_{+}\right\|_{p}\right)^{p}\right\}^{1 / p} .
\end{aligned}
$$

Proof. It suffices by Lemma 5.1 to show the following inequalities (these are valid for all $0<p<\infty$ ):

(i) $\inf _{u \in \mathscr{U}}\left\|x-u y u^{*}\right\|_{p} \leq\left\{\left|\left\|x_{+}\right\|_{p}-\left\|y_{+}\right\|_{p}\right|^{p}+\left|\left\|x_{-}\right\|_{p}-\left\|y_{-}\right\|_{p}\right|^{p}\right\}^{1 / p}$,

(ii) $\sup _{u \in \mathscr{U}}\left\|x-u y u^{*}\right\|_{p} \geq\left\{\left(\left\|x_{+}\right\|_{p}+\left\|y_{-}\right\|_{p}\right)^{p}+\left(\left\|x_{-}\right\|_{p}+\left\|y_{+}\right\|_{p}\right)^{p}\right\}^{1 / p}$.

By approximation, we may assume that $x_{+}, x_{-}, y_{+}$and $y_{-}$are all 
nonzero. From the assumption of $\mathscr{M}$, there exists a $v \in \mathscr{U}$ such that $s\left(x_{+}\right)=v s\left(y_{+}\right) v^{*}$ and $s\left(x_{-}\right)=v s\left(y_{-}\right) v^{*}$. So we can assume in proving (i) that there are projections $e$ and $f$ in $\mathscr{M}$ with $e \perp f$ such that $s\left(x_{+}\right)=s\left(y_{+}\right)=e$ and $s\left(x_{-}\right)=s\left(y_{-}\right)=f$. For each $u_{1} \in \mathscr{U}_{e}$ and $u_{2} \in \mathscr{U}_{f}$, let $u=u_{1}+u_{2}+(e+f)^{\perp}$. Then $u \in \mathscr{U}$ and

$$
\begin{aligned}
\left\|x-u y u^{*}\right\|_{p}^{p} & =\operatorname{tr}\left(\left|x-u y u^{*}\right|^{p}\right) \\
& =\operatorname{tr}\left(\left|x_{+}-u_{1} y_{+} u_{1}^{*}\right|^{p}+\left|x_{-}-u_{2} y_{-} u_{2}^{*}\right|^{p}\right) \\
& =\left\|x_{+}-u_{1} y_{+} u_{1}^{*}\right\|_{p}^{p}+\left\|x_{-}-u_{2} y_{-} u_{2}^{*}\right\|_{p}^{p} .
\end{aligned}
$$

Hence, by Lemma 5.2,

$$
\begin{aligned}
\inf _{u \in \mathscr{U}}\left\|x-u y u^{*}\right\|_{p}^{p} & \leq \inf _{u_{1} \in \mathscr{U}_{e}}\left\|x_{+}-u_{1} y_{+} u_{1}^{*}\right\|_{p}^{p}+\inf _{u_{2} \in \mathscr{U}_{f}}\left\|x_{-}-u_{2} y_{-} u_{2}^{*}\right\|_{p}^{p} \\
& \leq\left\|x_{+}-\frac{\left\|y_{+}\right\|_{p}}{\left\|x_{+}\right\|_{p}} x_{+}\right\|_{p}^{p}+\left\|x_{-}-\frac{\left\|y_{-}\right\|_{p}}{\left\|x_{-}\right\|_{p}} x_{-}\right\|_{p}^{p} \\
& =\left|\left\|x_{+}\right\|_{p}-\left\|y_{+}\right\|_{p}\right|^{p}+\left|\left\|x_{-}\right\|_{p}-\left\|y_{-}\right\|_{p}\right|^{p},
\end{aligned}
$$

so that we obtain (i). In proving (ii), we can assume as above that there are projections $e$ and $f$ in $\mathscr{M}$ with $e \perp f$ such that $s\left(x_{+}\right)=s\left(y_{-}\right)=e$ and $s\left(x_{-}\right)=s\left(y_{+}\right)=f$. Then, by Lemma 5.2 again,

$$
\begin{aligned}
\sup _{u \in \mathscr{U}}\left\|x-u y u^{*}\right\|_{p}^{p} & \geq \sup _{u_{1} \in \mathscr{U}_{e}}\left\|x_{+}+u_{1} y_{-} u_{1}^{*}\right\|_{p}^{p}+\sup _{u_{2} \in \mathscr{U}_{f}}\left\|x_{-}+u_{2} y_{+} u_{2}^{*}\right\|_{p}^{p} \\
& \geq\left\|x_{+}+\frac{\left\|y_{-}\right\|_{p}}{\left\|x_{+}\right\|_{p}} x_{+}\right\|_{p}^{p}+\left\|x_{-}+\frac{\left\|y_{+}\right\|_{p}}{\left\|x_{-}\right\|_{p}} x_{-}\right\|_{p}^{p} \\
& =\left(\left\|x_{+}\right\|_{p}+\left\|y_{-}\right\|_{p}\right)^{p}+\left(\left\|x_{-}\right\|_{p}+\left\|y_{+}\right\|_{p}\right)^{p},
\end{aligned}
$$

implying (ii).

Finally we obtain the formulas of $L^{p}$-distances for some classes of $x, y \in L^{p}(\mathscr{M})$ in the general infinite case, which are partial extensions of Theorems 4.4 (Remark 4.5(1)) and 4.6.

THEOREM 5.4. Assume that $\mathscr{M}$ is an arbitrary infinite factor.

(1) For every $x, y \in L^{p}(\mathscr{M})$ where $0<p \leq 1$,

$$
\sup _{u \in \mathscr{U}}\left\|x-u y u^{*}\right\|_{p}=\left(\|x\|_{p}^{p}+\|y\|_{p}^{p}\right)^{1 / p} .
$$

(2) For every $x, y \in L^{p}(\mathscr{M})+$ where $1 \leq p<\infty$,

$$
\begin{aligned}
& \sup _{u \in \mathscr{U}}\left\|x-u y u^{*}\right\|_{p}=\left(\|x\|_{p}^{p}+\|y\|_{p}^{p}\right)^{1 / p}, \\
& \inf _{u \in \mathscr{U}}\left\|x+u y u^{*}\right\|_{p}=\left(\|x\|_{p}^{p}+\|y\|_{p}^{p}\right)^{1 / p} .
\end{aligned}
$$


(3) For every $x, y \in L^{p}(\mathscr{M})_{s a}$ where $0<p \leq 2$,

$$
\sup _{u \in \mathscr{U}}\left\|x-i u y u^{*}\right\|_{p}=\left(\|x\|_{p}^{p}+\|y\|_{p}^{p}\right)^{1 / p} .
$$

(4) For every $x, y \in L^{p}(\mathscr{M})_{\text {sa }}$ where $2 \leq p<\infty$,

$$
\inf _{u \in \mathscr{U}}\left\|x-i u y u^{*}\right\|_{p}=\left(\|x\|_{p}^{p}+\|y\|_{p}^{p}\right)^{1 / p} .
$$

We give the next lemma to prove the theorem.

LEMMA 5.5. Assume that $\mathscr{M}$ is an arbitrary infinite factor. For every $x, y \in L^{p}(\mathscr{M})$ where $0<p<\infty$, there exist $x^{\prime}, y^{\prime} \in L^{p}(\mathscr{M})$ such that $x^{\prime}$ (resp. $\left.y^{\prime}\right)$ is in the $\|\cdot\|_{p}$-closure of $\mathscr{U}(x)($ resp. $\mathscr{U}(y))$ and $\left\|x^{\prime}-y^{\prime}\right\|_{p}=$ $\left(\|x\|_{p}^{p}+\|y\|_{p}^{p}\right)^{1 / p}$.

Proof. Let $0<p<\infty$ and $x, y \in L^{p}(\mathscr{M})$. Defining two projections $e=s(|x|) \vee s\left(\left|x^{*}\right|\right)$ and $f=s(|y|) \vee s\left(\left|y^{*}\right|\right)$ in $\mathscr{M}$, we choose projections $e^{\prime}$ and $f^{\prime}$ in $\mathscr{M}$ such that $e \sim e^{\prime}, f \sim f^{\prime}$ and $e^{\prime} \perp f^{\prime}$. Then $v^{*} v=$ $e, v v^{*}=e^{\prime}, w^{*} w=f$ and $w w^{*}=f^{\prime}$ for some partial isometries $v, w \in \mathscr{M}$. Since

$$
\|x\|_{p} \geq\left\|v x v^{*}\right\|_{p} \geq\|\operatorname{exe}\|_{p}=\|x\|_{p},
$$

$\left\|v x v^{*}\right\|_{p}=\|x\|_{p}$ and also $\left\|w y w^{*}\right\|_{p}=\|y\|_{p}$. Let $x^{\prime}=v x v^{*}$ and $y^{\prime}=$ $w y w^{*}$. Then

$$
\begin{aligned}
\left\|x^{\prime}-y^{\prime}\right\|_{p}^{p} & =\operatorname{tr}\left(\left|x^{\prime}-y^{\prime}\right|^{p}\right) \\
& =\operatorname{tr}\left(\left|x^{\prime}\right|^{p}+\left|y^{\prime}\right|^{p}\right)=\|x\|_{p}^{p}+\|y\|_{p}^{p} .
\end{aligned}
$$

Thus it suffices to show that $x^{\prime}$ (resp. $y^{\prime}$ ) is in the $\|\cdot\|_{p}$-closure of $\mathscr{U}(x)$ (resp. $\mathscr{U}(y))$. A sequence $\left\{e_{n}\right\}$ of projections in $\mathscr{M}$ can be chosen so that $e_{n} \nearrow e$ and $e_{n}^{\perp} \sim e_{n}^{\prime \perp}$ where $e_{n}^{\prime}=v e_{n} v^{*}$. For $n \geq 1$, let $v_{n}^{\prime}$ be a partial isometry in $\mathscr{M}$ such that $v_{n}^{\prime *} v_{n}^{\prime}=e_{n}^{\perp}$ and $v_{n}^{\prime} v_{n}^{\prime *}=e_{n}^{\prime \perp}$. Letting $u_{n}=v e_{n}+v_{n}^{\prime}$, we get $u_{n} \in \mathscr{U}$ and by [17, Theorem 4.9(iii)]

$$
\begin{aligned}
\left\|x^{\prime}-u_{n} x u_{n}^{*}\right\|_{p}^{p} & \leq 2^{p}\left\{\left\|v\left(x-e_{n} x e_{n}\right) v^{*}\right\|_{p}^{p}+\left\|u_{n}\left(e_{n} x e_{n}-x\right) u_{n}^{*}\right\|_{p}^{p}\right\} \\
& \leq 2^{p+1}\left\|x-e_{n} x e_{n}\right\|_{p}^{p} \\
& \leq 2^{2 p+1}\left(\left\|x-x e_{n}\right\|_{p}^{p}+\left\|x-e_{n} x\right\|_{p}^{p}\right) .
\end{aligned}
$$

When $1<p<\infty,\left\|x e_{n}\right\|_{p} \leq\|x\|_{p}$ and $x e_{n}$ converges weakly to $x$ since

$$
\left\langle x-x e_{n}, z\right\rangle=\operatorname{tr}\left(z x\left(e-e_{n}\right)\right) \rightarrow 0
$$

for every $z \in L^{q}(\mathscr{M})$ where $1 / p+1 / q=1$. Because $L^{p}(\mathscr{M})$ is uniformly convex (this is a consequence of Clarkson-McCarthy inequalities [17]), 
we have $\left\|x-x e_{n}\right\|_{p} \rightarrow 0$. When $0<p \leq 1$, choosing $\theta$ with $0<\theta<p$, we have by Hölder's inequality

$$
\begin{aligned}
\left\|x-x e_{n}\right\|_{p} & \leq\left\||x|\left(e-e_{n}\right)\right\|_{p} \\
& \leq\left\||x|^{1-\theta}\right\|_{p /(1-\theta)}\left\||x|^{\theta}\left(e-e_{n}\right)\right\|_{p / \theta} \\
& =\|x\|_{p}^{1-\theta}\left\||x|^{\theta}-|x|^{\theta} e_{n}\right\|_{p / \theta},
\end{aligned}
$$

so that $\left\|x-x e_{n}\right\|_{p} \rightarrow 0$ since $p / \theta>1$. Furthermore $\left\|x-e_{n} x\right\|_{p}=$ $\left\|x^{*}-x^{*} e_{n}\right\|_{p} \rightarrow 0$. Therefore $x^{\prime}$ is in the $\|\cdot\|_{p}$-closure of $\mathscr{U}(x)$. The assertion for $y^{\prime}$ is analogously shown.

Proof of Theorem 5.4. By Lemma 5.5, (1) and (2) follow from [17, Theorem 4.9(iii)] and Lemma 5.1, respectively. Let $x, y \in L^{p}(\mathscr{M})_{s a}$. Since $\|x+i y\|_{p}=\|x-i y\|_{p}$, we have by Clarkson-McCarthy inequalities [17]

$$
\begin{array}{ll}
\|x-i y\|_{p} \leq\left(\|x\|_{p}^{p}+\|y\|_{p}^{p}\right)^{1 / p}, & 1<p \leq 2, \\
\|x-i y\|_{p} \geq\left(\|x\|_{p}^{p}+\|y\|_{p}^{p}\right)^{1 / p}, & 2 \leq p<\infty .
\end{array}
$$

These and Lemma 5.5 imply (3) and (4).

When $\mathscr{M}$ is a factor of type $\mathrm{III}_{\lambda}, 0<\lambda<1$, we have another formulation of Haagerup $L^{p}$-spaces (discrete $L^{p}$-spaces) associated with the discrete decomposition of $\mathscr{M}$ (see $[19,25])$. We can exactly estimate the $L^{p}$-distance and the anti- $L^{p}$-distance between unitary orbits of $x, y \in L^{p}(\mathscr{M})_{s a}$ by using their spectral scales defined in the discrete $L^{p}$-space. Consequently, the diameter of the closed unitary orbits space in $\left\{x \in L^{p}(\mathscr{M})_{+}:\|x\|_{p}=1\right\}$ can be computed, including [12] as a special case. The details for the type $\mathrm{III}_{\lambda}$ case will be given in a forthcoming paper by the second named author.

Acknowledgment. We would like to thank Professor H. Kosaki for informing us of his generalization of the Powers-Størmer inequality and kindly writing it as the appendix of this paper.

Appendix. Generalized Powers-Størmer inequality by Hideki Kosaki (Department of Mathematics, College of General Education, Kyushu University, Fukuoka 810, Japan).

For positive compact operators $a, b$, the inequality

$$
\left\|a^{1 / 2}-b^{1 / 2}\right\|_{2} \leq\|a-b\|_{1}^{1 / 2}
$$

is known as the Powers-Størmer inequality, [37]. Here, $\|\cdot\|_{2}$ and $\|\cdot\|_{1}$ denote the Hilbert-Schmidt norm and the trace norm respectively. The 
same inequality for a general von Neumann algebra was obtained in [5] and [18], and it plays an important role in the theory of standard form. Similar inequalities in various set-ups have been investigated by many authors.

Let $L^{p}(\mathscr{M})$ be the Haagerup $L^{p}$-space described in $\S 5$. In this appendix we will prove the following generalization of the PowersStørmer inequality.

THEOREM. For positive $a, b$ in $L^{p}(\mathscr{M})$, we have

$$
\left\|a^{\theta}-b^{\theta}\right\|_{p / \theta} \leq\|a-b\|_{p}^{\theta}
$$

where $0<\theta<1$ and $\theta \leq p \leq \infty$.

In [2], Ando proved

$$
\begin{aligned}
\sum_{j=1}^{k} s_{j}(f(A)-f(B)) & \leq \sum_{j=1}^{k} s_{j}(f(|A-B|)) \\
& =\sum_{j=1}^{k} f\left(s_{j}(A-B)\right), \quad k=1,2, \ldots,
\end{aligned}
$$

for positive matrices $A, B$. Here, $s_{j}$ is the $j$ th largest eigenvalue and $f$ is an operator monotone function on $[0, \infty)$ satisfying $f(0)=0$ (see [16]). He began with the special case $A \geq B \geq 0$, that is,

$$
\sum_{j=1}^{k} s_{j}(f(B+C)-f(B)) \leq \sum_{j=1}^{k} s_{j}(f(C)), \quad k=1,2, \ldots,
$$

for positive matrices $B, C$. Replacing $s_{j}$ by the generalized $s$-number $\mu_{t}, t>0$ (see $\S 3$ ) and the partial sum by its continuous analogue $\int_{0}^{s} \cdot d t, s>0$, one can prove

$$
\int_{0}^{s} \mu_{t}(f(b+c)-f(b)) d t \leq \int_{0}^{s} \mu_{t}(f(c)) d t, \quad s>0,
$$

for positive operators $b, c$ in a semi-finite von Neumann algebra. Here exactly the same argument as in [2] works so that details are left to the reader. However, the following remark is in order: In [2], it is pointed out that the two matrices $(B+I)^{-1 / 2} C(B+I)^{-1 / 2}$ and $C^{1 / 2}(B+I)^{-1} C^{1 / 2}$ have the same lists of eigenvalues and consequently

$$
\begin{aligned}
& s_{j}\left(I-\left\{(B+I)^{-1 / 2} C(B+I)^{-1 / 2}+I\right\}^{-1}\right) \\
&= s_{j}\left(I-\left\{C^{1 / 2}(B+I)^{-1} C^{1 / 2}+I\right\}^{-1}\right) .
\end{aligned}
$$


In the present set-up, Lemma 2.5, [17], implies

$$
\begin{aligned}
\mu_{t}(1 & \left.-\left\{(b+1)^{-1 / 2} c(b+1)^{-1 / 2}+1\right\}^{-1}\right)=\mu_{t}\left(f_{0}\left(x x^{*}\right)\right) \\
& =f_{0}\left(\mu_{t}\left(x x^{*}\right)\right)=f_{0}\left(\mu_{t}\left(x^{*} x\right)\right)=\mu_{t}\left(f_{0}\left(x^{*} x\right)\right) \\
& =\mu_{t}\left(1-\left\{c^{1 / 2}(b+1)^{-1} c^{1 / 2}+1\right\}^{-1}\right),
\end{aligned}
$$

where $x=(b+1)^{-1 / 2} c^{1 / 2}$ and $f_{0}$ is the increasing function $1-(\lambda+1)^{-1}$ on $[0, \infty)$.

We then extend $(*)$ to (not necessarily bounded) $\tau$-measurable operators $b, c$. The original proof of this step was somewhat complicated. However, Professor Tikhonov kindly informed the author of his recent result saying that the map: $a \mapsto g(a)$ from a set of certain $\tau$-measurable operators is continuous with respect to the measure topology for a function $g$ in quite a wide class (Theorem 2.6, [43]). Using the spectral decomposition theorem, we choose two sequences $\left\{b_{n}\right\},\left\{c_{n}\right\}$ of positive elements in the von Neumann algebra satisfying $b_{n} \leq b, c_{n} \leq c$, and $b_{n} \rightarrow b, c_{n} \rightarrow c$ in measure. We have already known that

$$
\int_{0}^{s} \mu_{t}\left(f\left(b_{n}+c_{n}\right)-f\left(b_{n}\right)\right) d t \leq \int_{0}^{s} \mu_{t}\left(f\left(c_{n}\right)\right) d t, \quad s>0,
$$

for each $n$. By Tikhonov's result, $f\left(b_{n}+c_{n}\right)-f\left(b_{n}\right)$ converges to $f(b+c)-f(b)$ in measure so that

$$
\begin{aligned}
\int_{0}^{s} \mu_{t}(f(b+c)-f(b)) d t & \leq \int_{0}^{s} \liminf _{n \rightarrow \infty} \mu_{t}\left(f\left(b_{n}+c_{n}\right)-f\left(b_{n}\right)\right) d t \\
& (\text { Lemma 3.4, [17] }) \\
& \leq \liminf _{n \rightarrow \infty} \int_{0}^{s} \mu_{t}\left(f\left(b_{n}+c_{n}\right)-f\left(b_{n}\right)\right) d t .
\end{aligned}
$$

On the other hand, since $c_{n} \leq c$ and $f$ is operator monotone, we get

$$
\int_{0}^{s} \mu_{t}\left(f\left(c_{n}\right)\right) d t \leq \int_{0}^{s} \mu_{t}(f(c)) d t .
$$

Combining the above three estimates, we obtain $(*)$ for positive $\tau$ measurable operators $b, c$.

Now assume that $a$ and $b$ are generic positive $\tau$-measurable operators. Let $a-b=(a-b)_{+}-(a-b)_{-}$be the Jordan decomposition. Since $a \leq b+(a-b)_{+}$and $f$ is operator monotone, we know

$$
\begin{gathered}
f(a)-f(b) \leq f\left(b+(a-b)_{+}\right)-f(b), \\
(f(a)-f(b))_{+}=(f(a)-f(b)) e \leq e\left\{f\left(b+(a-b)_{+}\right)-f(b)\right\} e
\end{gathered}
$$


where $e$ is the support projection of $(f(a)-f(b))_{+}$. We hence get

$$
\begin{gathered}
\int_{0}^{s} \mu_{t}\left((f(a)-f(b))_{+}\right) d t \leq \int_{0}^{s} \mu_{t}\left(e\left\{f\left(b+(a-b)_{+}\right)-f(b)\right\} e\right) d t \\
\quad \leq \int_{0}^{s} \mu_{t}\left(f\left(b+(a-b)_{+}\right)-f(b)\right) d t \leq \int_{0}^{s} \mu_{t}\left(f\left((a-b)_{+}\right)\right) d t,
\end{gathered}
$$

applying $(*)$ to $b$ and $(a-b)_{+}$. The inequality for the negative parts can be obtained by changing the role of $a$ and $b$ in the preceding argument. Also, we can easily check (see $\S 3$ )

$$
\begin{aligned}
& \int_{0}^{s} \mu_{t}(f(a)-f(b)) d t \\
& =\sup _{0 \leq r \leq s}\left\{\int_{0}^{r} \mu_{t}\left((f(a)-f(b))_{+}\right) d t+\int_{0}^{s-r} \mu_{t}\left((f(a)-f(b))_{-}\right) d t\right\}, \\
& \int_{0}^{s} \mu_{t}(f(|a-b|)) d t \\
& \quad=\sup _{0 \leq r \leq s}\left\{\int_{0}^{r} \mu_{t}\left(f\left((a-b)_{+}\right)\right) d t+\int_{0}^{s-r} \mu_{t}\left(f\left((a-b)_{-}\right)\right) d t\right\} .
\end{aligned}
$$

Combining the above inequalities and equalities altogether, we obtain

$$
\begin{aligned}
\int_{0}^{s} \mu_{t}(f(a)-f(b)) d t & \leq \int_{0}^{s} \mu_{t}(f(|a-b|)) d t \\
& =\int_{0}^{s} f\left(\mu_{t}(a-b)\right) d t, \quad s>0,
\end{aligned}
$$

for positive $\tau$-measurable operators $a, b$.

If $\mathscr{M}$ is semi-finite, the desired generalization of the Powers-Størmer inequality follows from the above submajorization $(* *)$ as in [2]. However, we have to deal with a general von Neumann algebra and will make use of the trick repeatedly used in [30], [17] (and $\S 5$ of the main body of the article).

Finally let us prove the theorem. In the two extreme cases $p=\theta$ (Proposition 7, [30]) and $p=\infty$ (Theorem 2.3, [28]), the result is known. So let $\theta<p<\infty$. Assume that $a$ and $b$ are positive elements in $L^{p}(\mathscr{M})$. These are positive $\tau$-measurable operators affiliated with the crossed product $\mathscr{M} \rtimes_{\sigma^{\varphi_{0}}} \mathbf{R}$ ( $\tau$ is the canonical trace on the crossed product). Applying (**) (with $s=1$ and $f(\lambda)=\lambda^{\theta}$ ) to the semi-finite von Neumann algebra $\mathscr{M} \rtimes_{\sigma^{\varphi_{0}}} \mathbf{R}$, we get

$$
\int_{0}^{1} \mu_{t}\left(a^{\theta}-b^{\theta}\right) d t \leq \int_{0}^{1} \mu_{t}(a-b)^{\theta} d t
$$


where $\mu_{t}(\cdot)$ is relative to the canonical trace $\tau$. Recall (Lemma 4.8, [17]) that

$$
\begin{gathered}
\mu_{t}(a-b)=t^{-1 / p}\|a-b\|_{p}, \\
\mu_{t}\left(a^{\theta}-b^{\theta}\right)=t^{-\theta / p}\left\|a^{\theta}-b^{\theta}\right\|_{p / \theta} \quad\left(a^{\theta}, b^{\theta} \in L^{p / \theta}(\mathscr{M})\right),
\end{gathered}
$$

where $\|\cdot\|_{p}$ denotes the Haagerup (quasi-)norm. Thanks to $\theta<p$, we can explicitly evaluate the above two integrals and get

$$
(1-\theta / p)^{-1}\left\|a^{\theta}-b^{\theta}\right\|_{p / \theta} \leq(1-\theta / p)^{-1}\|a-b\|_{p}^{\theta},
$$

which proves the theorem.

\section{REFERENCES}

[1] T. Ando, Majorization, doubly stochastic matrices and comparison of eigenvalues, Lecture Notes, Hokkaido Univ., Sapporo, 1982; to appear in Linear Algebra Appl., (1989).

[2] - Comparison of norms $\||f(A)-f(B)|||$ and ||$|f(|A-B|)| \|$, Math. Z., 197 (1988), 403-409.

[3] T. Ando and R. Bhatia, Eigenvalue inequalities associated with the Cartesian decomposition, Linear and Multilinear Algebra, 22 (1987), 133-147.

[4] T. Ando and Y. Nakamura, Anti-distance between unitary orbits of operators, unpublished notes, 1986.

[5] H. Araki, Some properties of modular conjugation operator of von Neumann algebras and a non-commutative Radon-Nikodym theorem with a chain rule, Pacific J. Math., 50 (1974), 309-354.

[6] E. A. Azoff and C. Davis, On distances between unitary orbits of self-adjoint operators, Acta Sci. Math., 47 (1984), 419-439.

[7] R. Bhatia and C. Davis, A bound for the spectral variation of a unitary operator, Linear and Multilinear Algebra, 15 (1984), 71-76.

[8] R. Bhatia, C. Davis and P. Koosis, An extremal problem in Fourier analysis with applications to operator theory, J. Funct. Anal., 82 (1989), 138-150.

[9] R. Bhatia, C. Davis and A. McIntosh, Perturbations of spectral subspaces and solution of linear operator equations, Linear Algebra Appl., 52/53 (1983), 45-67.

[10] R. Bhatia and J. A. R. Holbrook, Short normal paths and spectral variation, Proc. Amer. Math. Soc., 94 (1985), 377-382.

[11] K. M. Chong, Some extensions of a theorem of Hardy, Littlewood and Pólya and their applications, Canad. J. Math., 26 (1974), 1321-1340.

[12] A. Connes, U. Haagerup and E. Størmer, Diameters of state spaces of type III factors, Operator Algebras and their Connections with Topology and Ergodic Theory (H. Araki et al. eds.), Lecture Notes in Math., No. 1132, Springer-Verlag, Berlin, 1985, pp. 91-116.

[13] A. Connes and E. Størmer, Homogeneity of the state space of factors of type $\mathrm{III}_{1}$, J. Funct. Anal., 28 (1978), 187-196.

[14] K. R. Davidson, The distance between unitary orbits of normal operators, Acta Sci. Math., 50 (1986), 213-223.

[15] J. Dixmier, Formes linéaires sur un anneau d'opérateurs, Bull. Soc. Math. France, 81 (1953), 9-39. 
[16] W. Donoghue, Monotone Matrix Functions and Analytic Continuation, SpringerVerlag, Berlin-Heidelberg-New York, 1974.

[17] T. Fack and H. Kosaki, Generalized s-numbers of $\tau$-measurable operators, Pacific J. Math., 123 (1986), 269-300.

[18] U. Haagerup, The standard form of von Neumann algebras, Math. Scand., 37 (1975), 271-283.

[19],$L^{p}$-spaces associated with an arbitrary von Neumann algebra, Colloq. Internat. CNRS, No. 274, 1979, pp. 175-184.

[20] P. Hall, On représentatives of subsets, J. London Math. Soc., 10 (1935), 26-30.

[21] R. H. Herman and A. Ocneanu, Spectral analysis for automorphisms of UHF $C^{*}$-algebras, J. Funct. Anal., 66 (1986), 1-10.

[22] F. Hiai, Majorization and stochastic maps in von Neumann algebras, J. Math. Anal. Appl., 127 (1987), 18-48.

[23] __ Spectral relations and unitary mixing in semifinite von Neumann algebras, Hokkaido Math. J., 17 (1988), 117-137.

[24] F. Hiai and Y. Nakamura, Majorizations for generalized s-numbers in semifinite von Neumann algebras, Math. Z., 195 (1987), 17-27.

[25] V. Kaftal and R. Mercer, Spectral projections of $L^{1}$ operators in type $\mathrm{III}_{\lambda}$ von Neumann algebras, Integral Equations Operator Theory, 9 (1986), 679-693.

[26] E. Kamei, Double stochasticity in finite factors, Math. Japon., 29 (1984), 903907.

[27] _ An order on statistical operators implicitly introduced by von Neumann, Math. Japon., 30 (1985), 891-895.

[28] F. Kittaneh and H. Kosaki, Inequalities for the Schatten p-norm V, Publ. RIMS, Kyoto Univ., 23 (1987), 433-443.

[29] H. Kosaki, Applications of uniform convexity of noncommutative $L^{p}$-spaces, Trans. Amer. Math. Soc., 283 (1984), 265-282.

[30] On the continuity of the map $\varphi \rightarrow|\varphi|$ from the predual of a $W^{*}$-algebra, J. Funct. Anal., 59 (1984), 123-131.

[31] A. S. Markus, The eigen- and singular values of the sum and product of linear operators, Russian Math. Surveys, 19 (1964), 91-120.

[32] A. W. Marshall and I. Olkin, Inequalities: Theory of Majorization and Its Applications, Academic Press, New York, 1979.

[33] Y. Nakamura, An inequality for generalized s-numbers, Integral Equations Operator Theory, 10 (1987), 140-145.

[34] E. Nelson, Notes on non-commutative integration, J. Funct. Anal., 15 (1974), 103-116.

[35] V. I. Ovchinnikov, s-numbers of measurable operators, Functional Anal. Appl., 4 (1970), 236-242.

[36] D. Petz, Spectral scale of self-adjoint operators and trace inequalities, J. Math. Anal. Appl., 109 (1985), 74-82.

[37] R. T. Powers and E. Størmer, Free states of the canonical anticommutation relations, Comm. Math. Phys., 16 (1970), 1-33.

[38] Y. Sakai, Weak spectral order of Hardy, Littlewood and Pólya, J. Math. Anal. Appl., 108 (1985), 31-46.

[39] I. Segal, A non-commutative extension of abstract integration, Ann. of Math., 57 (1953), 401-457.

[40] E. M. Stein and G. Weiss, Introduction of Fourier Analysis on Euclidean Spaces, Princeton Univ. Press, Princeton, 1971.

[41] V. S. Sunder, Distance between normal operators, Proc. Amer. Math. Soc., 84 (1982), 483-484. 
[42] M. Terp, $L^{p}$ spaces associated with von Neumann algebras, Notes, Copenhagen Univ., 1981.

[43] O. E. Tikhonov, Continuity of operator functions in topologies connected with a trace on a von Neumann algebra (Russian), Izv. Vyssh. Uchebn. Zaved. Mat., 1987, no. 1, 77-79; translated in Soviet Math. (Iz. VUZ), 31 (1987), 110-114.

[44] H. Umegaki, Conditional expectation in an operator algebra, I, II, III, IV, Tôhoku Math. J., 6 (1954), 177-181; ibid., 8 (1956), 86-100; Kōdai Math. Sem. Rep., 11 (1959), 51-64; ibid., 14 (1962), 59-85.

[45] H. Weyl, Das asymptotische Verteilungsgesetz der Eigenwerte linearer partieller Differentialgleichungen, Math. Ann., 71 (1912), 441-479.

[46] F. J. Yeadon, Non-commutative $L^{p}$-spaces, Math. Proc. Cambridge Philos. Soc., 77 (1975), 91-102.

Received July 15, 1987 and in revised form July 6, 1988. The research by the authors was supported, in part, by Grant-in-Aid for Scientific Research.

Division of Applied Mathematics

RESEARCH INSTITUTE OF APPLIED ELECTRICITY

HOKKAIDO UNIVERSITY

SAPPORO 060, JAPAN 